\title{
HYPERBOLIC RELAXATION MODELS FOR GRANULAR FLOWS
}

\author{
Thierry Gallouët ${ }^{1}$, Philippe Helluy ${ }^{1}$, Jean-Marc Hérard $^{1}$ \\ AND Julien Nussbaum ${ }^{1}$
}

\begin{abstract}
In this work we describe an efficient model for the simulation of a two-phase flow made of a gas and a granular solid. The starting point is the two-velocity two-pressure model of Baer and Nunziato [Int. J. Multiph. Flow 16 (1986) 861-889]. The model is supplemented by a relaxation source term in order to take into account the pressure equilibrium between the two phases and the granular stress in the solid phase. We show that the relaxation process can be made thermodynamically coherent with an adequate choice of the granular stress. We then propose a numerical scheme based on a splitting approach. Each step of the time marching algorithm is made of two stages. In the first stage, the homogeneous convection equations are solved by a standard finite volume Rusanov scheme. In the second stage, the volume fraction is updated in order to take into account the equilibrium source term. The whole procedure is entropy dissipative. For simplified pressure laws (stiffened gas laws) we are able to prove that the approximated volume fraction stays within its natural bounds.
\end{abstract}

Mathematics Subject Classification. 76M12, 65M12.

Received June 19, 2008. Revised July 9, 2009.

Published online January 27, 2010.

\section{INTRODUCTION}

We are interested in the numerical modelling of a two-phase (granular-gas) flow with two velocities and two pressures $p_{1}$ and $p_{2}$. In one space dimension, the model is made up of seven non-homogeneous partial differential equations: two mass balance laws, two momentum balance laws, two energy balance laws and one volume fraction evolution equation. It is similar to the initial model proposed by Baer and Nunziato [1]. The main feature of this model is that the left hand side of the equations is hyperbolic. This property is very important because it ensures the mathematical stability of the model.

However, in many industrial applications it is not realistic to admit two independent pressures. Generally, an algebraic relation between the two pressures is assumed. An example (among many others) of such a modelling in the framework of internal ballistics is given by Gough in [8]. For a general presentation of two-phase flow models, we refer to the book of Gidaspow [5]. The relation between the two pressures is classically of the form $p_{2}=p_{1}+R$ where $R$ is the granular stress. In the general case, the granular stress depends on all the thermodynamic variables of the two phases.

Keywords and phrases. Two-phase flow, hyperbolicity, relaxation, finite volume, entropy.

1 IRMA, 7 rue Descartes, 67084 Strasbourg Cedex, France. helluy@math.u-strasbg.fr 
Because of the pressure relation, the system is now overdetermined. The volume fraction equation can be eliminated and a six-equation model is obtained. Unfortunately, the new model has a reduced hyperbolicity domain. The worst situation corresponds to a vanishing granular stress $R=0$. In this case, the model is almost never hyperbolic.

In the case of a vanishing granular stress, several authors have proposed to relax the algebraic relation $p_{2}=p_{1}$ by adding an adequate source term to the volume fraction evolution $[4,11,17,18]$, etc. An important parameter of the source term is the characteristic equilibrium time. When the equilibration time tends to zero, the six equation model is recovered. When the equilibrium time does not vanish the stability of the model is expected.

In this paper, we extend the relaxation approach to non-vanishing granular stress $R>0$. With a positive granular stress, the hyperbolicity domain of the six-equation model is slightly extended. But it is generally not possible to remove all the elliptic regions. Therefore, we apply a relaxation source term to the volume fraction evolution equation of the seven-equation model. This source term takes into account the granular stress (see (2.12)). When the relaxation parameter $\tau_{p}$ tends to 0 , we recover the equilibrium six-equation model. When the relaxation parameter $\tau_{p}>0$, the stability of the model is recovered.

An important aspect of the model is that the granular stress cannot be chosen arbitrarily once the pressure law of the solid phase is fixed. Indeed, it has to satisfy some thermodynamical relations in order that an entropy dissipation equation can be established. We illustrate this fact when the equation of state of the solid phase is a stiffened gas law. We propose a very simple but useful expression (see (3.11)) for the granular stress, which is mathematically and physically relevant.

Then we propose a numerical method to solve the relaxed system. For that purpose, we use a splitting algorithm. Each time step of the algorithm consists in:

- evolving the seven equation model without the source term;

- solving the relaxed pressure equilibrium with granular stress;

- solving the other source terms.

In the second stage the relaxed pressure equilibrium implies to solve an update for the volume fraction, keeping the conserved variables constant. Under some monotony hypothesis on the granular stress, and when the pressure laws of the two phases are stiffened gas laws, we are able to prove the existence and uniqueness of the new volume fraction in the interval ] $0,1[$.

The relaxed equilibrium becomes an exact equilibrium when the relaxation parameter $\tau_{p}=0$. Thus our method can also be used to approximate the equilibrium six-equation model on coarse meshes, which is important in industrial applications.

Finally, we propose some numerical experiments. For academic test cases, we highlight some behavior of the relaxed approach in the case of a non-stable (elliptic) case. We then compare the results of our new approach with the standard Gough model $[8,15]$ in the case of a simplified internal ballistics problem.

\section{Notations AND MODEL}

We consider a two-phase flow of a granular solid mixed with a compressible gas. The solid is denoted by the index (2) and the gas by the index (1). For more generality, the solid is supposed to be compressible. The unknowns are, for each phase $k=1,2$, the partial density $\rho_{k}$, the velocity $u_{k}$, the internal energy $e_{k}$. The volume fractions $\alpha_{k}$ satisfy $\alpha_{1}+\alpha_{2}=1$. The gas volume fraction $\alpha_{1}$ is also called the porosity in the context of granular flows. The pressure of each phase is given by an equation of state of the form

$$
p_{k}=\psi_{k}\left(\rho_{k}, e_{k}\right) \text {. }
$$

We note $\alpha_{k} \rho_{k}=m_{k}$. The principles of mass, momentum and energy conservation imply that some source terms will cancel. Thus, we introduce the following notation for the sign function

$$
\sigma_{k}:=\left\{\begin{array}{l}
+1 \text { if } k=1 \\
-1 \text { if } k=2
\end{array}\right.
$$


The balance of mass, momentum and energy read

$$
\begin{aligned}
& m_{k, t}+\left(m_{k} u_{k}\right)_{x}=\sigma_{k} M, \\
& \left(m_{k} u_{k}\right)_{t}+\left(m_{k} u_{k}^{2}+\alpha_{k} p_{k}\right)_{x}-p_{I} \alpha_{k, x}=\sigma_{k} Q, \\
& \left(m_{k} E_{k}\right)_{t}+\left(\left(m_{k} E_{k}+\alpha_{k} p_{k}\right) u_{k}\right)_{x}+p_{I} \alpha_{k, t}=\sigma_{k} S, \\
& \alpha_{k, t}+v_{I} \alpha_{k, x}=\sigma_{k} P,
\end{aligned}
$$

where

$$
E_{k}=e_{k}+\frac{u_{k}^{2}}{2}
$$

For simplicity, we restrict ourselves to one-dimensional equations, but it is possible to extend the results of this paper to higher space dimensions. The right hand side terms $M, Q, P, S$ are internal exchange source terms that will be discussed later. For the moment, introducing the vector of the seven main unknowns of the system (1.3)

$$
W=\left(m_{1}, m_{1} u_{1}, m_{1} E_{1}, m_{2}, m_{2} u_{2}, m_{2} E_{2}, \alpha_{1}\right)^{T}
$$

we only suppose that $M, Q, P, S$ are general functions of $W$. Here, we recall that the $\operatorname{sign} \sigma_{k}=+1$ if $k=1$ and $\sigma_{k}=-1$ if $k=2$. We also suppose that there are no external force and energy source (this explains the $\sigma_{k}$ signs in the source terms). The quantities $p_{I}$ and $v_{I}$ are respectively the interface pressure and the interface velocity. In this paper, we take the special choice of Baer-Nunziato

$$
\begin{aligned}
p_{I} & =p_{1}, \\
v_{I} & =u_{2} .
\end{aligned}
$$

This model for the interface pressure and velocity is physically relevant when the solid phase is dilute in the gas-solid mixture (see [1]). Of course, due to the perfect symmetry of the PDE system with respect to the phase index $k$, a symmetric choice is also possible

$$
\begin{aligned}
& p_{I}=p_{2}, \\
& v_{I}=u_{1},
\end{aligned}
$$

when the solid phase is packed. It is also possible to consider interface pressure and velocity that are convex linear combinations of the phase pressures and velocities [3,4,17]. Quite surprisingly, the convex linear combination is not generally considered as the good physical modelling.

In this paper, we focus on the dilute case (1.6) which enjoys good properties ${ }^{2}$ :

- the left hand side of the system is hyperbolic (the proof is recalled in Sect. A);

- this choice ensures that the non-conservative products are well defined (at least in the non-resonant case). This is due to the fact that the volume fraction only jumps in the linearly degenerated field associated to the eigenvalue $v_{I}$. In a linearly degenerated field, the jump relations are simply provided by the Riemann invariants of this field. See [3,4];

- in the applications, the Baer-Nunziato model is particularly adapted to granular flows. See [1] and included references.

Using the volume fraction equation, the time derivative $\alpha_{k, t}$ can be replaced by space derivatives

$$
\begin{aligned}
& m_{k, t}+\left(m_{k} u_{k}\right)_{x}=\sigma_{k} M, \\
& \left(m_{k} u_{k}\right)_{t}+\left(m_{k} u_{k}^{2}\right)_{x}+\alpha_{k} p_{k, x}+\left(p_{k}-p_{I}\right) \alpha_{k, x}=\sigma_{k} Q, \\
& \left(m_{k} E_{k}\right)_{t}+\left(m_{k} E_{k} u_{k}\right)_{x}+\alpha_{k}\left(p_{k} u_{k}\right)_{x}+\left(p_{k} u_{k}-p_{I} v_{I}\right) \alpha_{k, x}=\sigma_{k} S-\sigma_{k} p_{I} P, \\
& \alpha_{k, t}+v_{I} \alpha_{k, x}=\sigma_{k} P .
\end{aligned}
$$

\footnotetext{
${ }^{2}$ The packed model (1.7) and the convex combination models given in [4] enjoy the same properties.
} 
The equations can then be written under the form of a first order non-conservative and non-homogeneous system

with

$$
W_{t}+F(W)_{x}+A(W) L(W)_{x}=\Sigma(W)
$$

$$
\begin{aligned}
& W=\left(m_{1}, m_{1} u_{1}, m_{1} E_{1}, m_{2}, m_{2} u_{2}, m_{2} E_{2}, \alpha_{1}\right)^{T}, \\
& F(W)=\left(m_{1} u_{1}, m_{1} u_{1}^{2}, m_{1} E_{1} u_{1}, m_{2} u_{2}, m_{2} u_{2}^{2}, m_{2} E_{2} u_{2}, 0\right)^{T}, \\
& L(W)=\left(p_{1}, p_{1} u_{1}, p_{2}, p_{2} u_{2}, \alpha_{1}\right)^{T}, \\
& A(W) L(W)_{x}=\left(0, \alpha_{1} p_{1, x}, \alpha_{1}\left(p_{1} u_{1}\right)_{x}+p_{1}\left(u_{1}-u_{2}\right) \alpha_{1, x}, 0, \alpha_{2} p_{2, x}\right. \\
& \left.+\left(p_{2}-p_{1}\right) \alpha_{2, x}, \alpha_{2}\left(p_{2} u_{2}\right)_{x}+u_{2}\left(p_{2}-p_{1}\right) \alpha_{2, x}, v_{2} \alpha_{1, x}\right)^{T}, \\
& \Sigma(W)=\left(M(W), Q(W), S(W)-p_{1} P(W),\right. \\
& \left.-M(W),-Q(W),-S(W)+p_{1} P(W), P(W)\right)^{T} .
\end{aligned}
$$

Let us note that this writing is not unique. We have chosen a formulation in which the non-conservative terms vanish when the pressures and velocities are constant, i.e.

$$
p_{1}=p_{2}=p_{0}=\text { Cst. and } u_{1}=u_{2}=u_{0}=\text { Cst. }
$$

This particular representation is useful to design numerical schemes that perfectly preserve states where the velocity and the pressure are constant (see the definitions (4.2), (4.3)).

\section{ENTROPY DISSIPATION}

In this section, we establish an entropy dissipation equation. This equation is very important because it permits to select the source terms that are compatible with the second principle of thermodynamics.

For that purpose we first rewrite the system as follows

$$
\begin{aligned}
& m_{k}\left(u_{k, t}+u_{k} u_{k, x}\right)+\left(\alpha_{k} p_{k}\right)_{x}-p_{1} \alpha_{k, x}=\sigma_{k} Q-\sigma_{k} u_{k} M, m_{k}\left(e_{k, t}+u_{k} e_{k, x}\right) \\
& \quad+\frac{u_{k}}{2}\left[\left(m_{k} u_{k}\right)_{t}+\left(m_{k} u_{k}^{2}+\alpha_{k} p_{k}\right)_{x}+\left(\alpha_{k} p_{k}\right)_{x}\right]+\frac{1}{2} m_{k} u_{k}\left(u_{k, t}+u_{k} u_{k, x}\right)+\alpha_{k} p_{k} u_{k, x}+p_{1} \alpha_{k, t}=\sigma_{k} S-\sigma_{k} e_{k} M .
\end{aligned}
$$

The last equation also reads

$$
\begin{aligned}
m_{k}\left(e_{k, t}+u_{k} e_{k, x}\right)+\frac{u_{k}}{2}\left[p_{1} \alpha_{k, x} \sigma_{k} Q+\left(\alpha_{k} p_{k}\right)_{x}\right]+\frac{1}{2} u_{k}\left(-\left(\alpha_{k} p_{k}\right)_{x}+\right. & \left.p_{1} \alpha_{k, x} \sigma_{k} Q-\sigma_{k} u_{k} M\right) \\
& +\alpha_{k} p_{k} u_{k, x}+p_{1} \alpha_{k, t}=\sigma_{k} S-\sigma_{k} e_{k} M
\end{aligned}
$$

and

$$
m_{k}\left(e_{k, t}+u_{k} e_{k, x}\right)+\alpha_{k} p_{k} u_{k, x}+p_{1}\left(u_{k}-u_{2}\right) \alpha_{k, x}=\sigma_{k} S-\sigma_{k} e_{k} M-\sigma_{k} u_{k} Q \sigma_{k} \frac{1}{2} u_{k}^{2} M-\sigma_{k} p_{1} P .
$$

Finally, we obtain

$$
\begin{aligned}
& \alpha_{k, t}+u_{2} \alpha_{k, x}=\sigma_{k} P \\
& \alpha_{k}\left(\rho_{k, t}+u_{k} \rho_{k, x}\right)+\rho_{k}\left(u_{k}-u_{2}\right) \alpha_{k, x}+m_{k} u_{k, x}=\sigma_{k} M-\sigma_{k} \rho_{k} P \\
& m_{k}\left(u_{k, t}+u_{k} u_{k, x}\right)+\left(\alpha_{k} p_{k}\right) x-p_{1} \alpha_{k, x}=\sigma_{k} Q-\sigma_{k} u_{k} M, \\
& m_{k}\left(e_{k, t}+u_{k} e_{k, x}\right)+\alpha_{k} p_{k} u_{k, x}+p_{1}\left(u_{k}-u_{2}\right) \alpha_{k, x}=\sigma_{k} S-\sigma_{k} e_{k} M-\sigma_{k} u_{k} Q \sigma_{k} \frac{1}{2} u_{k}^{2} M-\sigma_{k} p_{1} P .
\end{aligned}
$$


Now, we introduce entropies $s_{k}$ for the two phases. The entropy of the phase $k=1,2$ satisfies the following first order partial differential equation

$$
\begin{aligned}
T_{k} \mathrm{~d} s_{k} & =\mathrm{d} e_{k}-\frac{p_{k}}{\rho_{k}^{2}} \mathrm{~d} \rho_{k}-\frac{R_{k}}{m_{k}} \mathrm{~d} \alpha_{k} \\
& =T_{k}\left(\frac{\partial s_{k}}{\partial e_{k}}\right) \mathrm{d} e_{k}+T_{k}\left(\frac{\partial s_{k}}{\partial \rho_{k}}\right) \mathrm{d} \rho_{k}+T_{k}\left(\frac{\partial s_{k}}{\partial \alpha_{k}}\right) \mathrm{d} \alpha_{k} .
\end{aligned}
$$

The temperature of phase $k$ is denoted by $T_{k}$. The granular stress in the phase $k$ is denoted by $R_{k}$. Physically, the term $-R_{k} / m_{k} \mathrm{~d} \alpha_{k}$ represents the work of the granular stress due to a change of volume $\mathrm{d} \alpha_{k}$. For sake of simplicity and in order to be more realistic, we assume that the "granular" stress $R_{1}$ vanishes in the gas phase, thus $R_{1}=0$. Without ambiguity, we can also denote the granular stress in the solid phase by $R=R_{2}$. Generally, the granular stress $R$ depends on $\left(\rho_{2}, e_{2}, \alpha_{2}\right)$. A more precise formulation of the granular stress $R$ is discussed in Section 3.

The chemical potential of phase $k$ is noted $\mu_{k}$ and is defined by

$$
\mu_{k}=e_{k}+\frac{p_{k}}{\rho_{k}}-T_{k} s_{k}
$$

We multiply the last equation in (2.4) by $1 / T_{k}$, the second by $-p_{k} / \rho_{k} / T_{k}$, the first by $-R_{k} / T_{k}$ and take the sum

$$
\begin{aligned}
& m_{k}\left(s_{k, t}+u_{k} s_{k, x}\right)+\frac{p_{1}-p_{k}}{T_{k}}\left(u_{k}-u_{2}\right) \alpha_{k, x}=\frac{1}{T_{k}}\left(\sigma_{k} P\left(-R_{k}+p_{k}-p_{1}\right) \sigma_{k} M\left(\frac{u_{k}^{2}}{2}-\frac{p_{k}}{\rho_{k}}-e_{k}\right) \sigma_{k} Q\left(-u_{k}\right) \sigma_{k} S\right) \\
& \left(m_{k} s_{k}\right)_{t}+\left(m_{k} u_{k} s_{k}\right)_{x}=\frac{1}{T_{k}}\left(\sigma_{k} P\left(p_{k}-R_{k}-p_{1}\right) \sigma_{k} M\left(T_{k} s_{k}+\frac{u_{k}^{2}}{2}-\frac{p_{k}}{\rho_{k}}-e_{k}\right) \sigma_{k} Q\left(-u_{k}\right) \sigma_{k} S\right) .
\end{aligned}
$$

Adding now the two entropy equations leads to the entropy dissipation partial differential equation that we sum up in the following proposition.

Proposition 2.1. Consider a smooth solution of the system (1.3) and two entropy functions $s_{1}$ and $s_{2}$ satisfying (2.5). Then, the smooth solution satisfies the following entropy dissipation equation

$$
\begin{aligned}
\left(\sum m_{k} s_{k}\right)_{t}+\left(\sum m_{k} u_{k} s_{k}\right)_{x}=\frac{P}{T_{2}}\left(p_{1}+R-p_{2}\right)+M\left(\frac{u_{1}^{2}}{2 T_{1}}-\frac{u_{2}^{2}}{2 T_{2}}-\frac{\mu_{1}}{T_{1}}+\frac{\mu_{2}}{T_{2}}\right) & \\
& +Q\left(\frac{u_{2}}{T_{2}}-\frac{u_{1}}{T_{1}}\right)+S\left(\frac{1}{T_{1}}-\frac{1}{T_{2}}\right) .
\end{aligned}
$$

Remark 2.1. According to the second principle of thermodynamics the right hand side of (2.8) has to be non-negative. But each term in the formula (2.8) has not a clear physical meaning and the Galilean invariance is not obvious. It is often more convenient to rewrite the source term in a different way. For example, we can set

$$
\begin{aligned}
& Q=Q_{0}+u_{1} M, \\
& S=S_{0}+u_{1} Q_{0}+\frac{u_{1}^{2}}{2} M+\mu_{1} M .
\end{aligned}
$$

In this way, the dissipation rate becomes

$$
\frac{P}{T_{2}}\left(p_{1}+R-p_{2}\right)+\frac{M}{T_{2}}\left(\mu_{2}-\mu_{1}-\frac{\left(u_{2}-u_{1}\right)^{2}}{2}\right)+\frac{Q_{0}}{T_{2}}\left(u_{2}-u_{1}\right)+\frac{S_{0}}{T_{1} T_{2}}\left(T_{2}-T_{1}\right) .
$$

It is $>0$ if each term in the sum is $>0$. The source $S_{0}$ can then be interpreted as the heat flux (it is $>0$ when $T_{2}>T_{1}$, i.e. when the phase 2 heats the phase 1 ). The source $Q_{0}$ is the drag force. Finally, $M$ is the mass 
transfer due to chemical reactions. When $u_{1}=u_{2}$, we recover that the chemical reaction tends to create the phase with the smallest chemical potential.

Remark 2.2. Generally, equations (2.5) satisfied by the entropies $s_{k}$ have not a unique solution, once the pressure laws are given. For example, if $s_{k}$ is a solution, $-s_{k}$ is also a solution. A supplementary condition has thus to be given in order to fix the sign of the entropy dissipation rate. In the case of conservative systems the entropies are supposed to satisfy some convexity property. For a non-conservative system, it is not possible to apply the Godunov-Mock theorem and it is difficult to extend naturally the convexity approach. We propose here only to forbid the change $s \rightarrow-s$ by imposing that the temperature remains $>0$. It implies

$$
\frac{1}{T_{k}}=\frac{\partial s_{k}}{\partial e_{k}}>0
$$

In this paper, we will concentrate on the pressure relaxation source term. We will assume the following form, which ensures a positive entropy dissipation

$$
P=\frac{1}{\tau_{p}} \alpha_{1} \alpha_{2}\left(p_{1}+R-p_{2}\right), \quad \tau_{p}>0
$$

where $\tau_{p}$ is the relaxation parameter. An instantaneous relaxation corresponds to the limit $\tau_{p}=0$.

Remark 2.3. Let $p_{\text {ref }}$ be a reference pressure. We can define a characteristic time for the pressure equilibrium by

$$
t_{\mathrm{ref}}=\frac{\tau_{p}}{p_{\mathrm{ref}}}
$$

The knowledge of this characteristic time is important for a proper modelling.

\section{Application to STIFFEnEd GAS LAWS: ADMissible GRANUlAR STRESS}

\subsection{Admissible granular stress}

In many works (as in $[7,12,13]$ ), the granular stress is supposed to depend only on the solid volume fraction $\alpha_{2}$. This hypothesis is reasonable when the solid phase is incompressible. However, this choice is not compatible with the existence of an entropy satisfying (2.5) in the case of a compressible phase. The choice of the granular stress expression cannot be arbitrary. That is why in this section, we compute a simple expression of it when the pressure law of the solid phase is a stiffened gas equation of state.

Let us note

$$
\Theta\left(1 / \rho_{2}, e, \alpha_{2}\right)=\frac{R_{2}\left(1 / \rho_{2}, e, \alpha_{2}\right)}{\alpha_{2} \rho_{2}} .
$$

We omit now the subscript $k=2$ because we concentrate only on the solid phase. We have to find an entropy $s$, a temperature $T$ and a function $\Theta$ (containing the granular modelling) such that

$$
T \mathrm{~d} s=\mathrm{d} e-\frac{p}{\rho^{2}} \mathrm{~d} \rho-\Theta \mathrm{d} \alpha
$$

We note

$$
\begin{aligned}
& \tau=1 / \rho, \\
& T=1 / \varphi .
\end{aligned}
$$

Then, $\varphi=\varphi(\tau, e, \alpha)$ is an integrating factor for the form

$$
\mathrm{d} e+p \mathrm{~d} \tau-\Theta \mathrm{d} \alpha
$$


which reads

$$
\mathrm{d} s=\varphi \mathrm{d} e+\varphi p(\tau, e) \mathrm{d} \tau-\varphi \Theta \mathrm{d} \alpha .
$$

In order to construct a practical and simple model, we suppose that the granular stress only depends on the density and the volume fraction of the solid phase. This leads to the choice $\Theta=\Theta(\tau, \alpha)$. The differential form is closed if

$$
\begin{aligned}
& \varphi_{\alpha}=-\Theta \varphi_{e}, \\
& p \varphi_{\alpha}=-\Theta \varphi_{\tau}-\varphi \Theta_{\tau}, \\
& p \varphi_{e}+p_{e} \varphi=\varphi_{\tau} .
\end{aligned}
$$

The general case corresponds to $\varphi_{e} \neq 0, \varphi_{\alpha} \neq 0$ and $\varphi \neq 0$. We then have necessarily

$$
\frac{\Theta_{\tau}}{\Theta}=-p_{e}
$$

\subsection{Practical example}

Now we propose some computations when the pressure law (1.1) is a stiffened gas EOS

$$
p=\psi(\rho, e)=(\gamma-1) \rho e-\gamma \pi
$$

The parameter $\gamma$ must be $>1$. The parameter $\pi$ has the dimension of a pressure and can be arbitrary. But in practice, for a solid phase, it is positive and large compared to a characteristic pressure of the flow. In the case of a stiffened gas equation, we thus find

$$
\frac{\Theta_{\tau}}{\Theta}=-\frac{\gamma-1}{\tau} \Rightarrow \Theta(1 / \rho, \alpha)=\theta(\alpha) \rho^{\gamma-1}
$$

which leads to

$$
R(\tau, \alpha)=\alpha \theta(\alpha) \rho^{\gamma} .
$$

In this paper, we will perform numerical experiments with a very simple particular choice

$$
R(\tau, \alpha)=\kappa \rho^{\gamma} \alpha^{\gamma}
$$

The value of $\kappa$ can be adjusted to experiments. With this choice, the parameter $R_{0}=\kappa \rho_{2}^{\gamma_{2}}$ has the dimension of a pressure. It represents the maximal stress corresponding to the maximal compaction $\alpha=\alpha_{2}=1$. This model is not so different from classical approaches (as described for example in [7]): usually, the granular stress vanishes under some critical solid volume fraction $\alpha_{c}$ (dilute case) and increases with $\alpha$ when $\alpha>\alpha_{c}$ (packed case). In our approach, the parameter $\gamma$ allows to ensure that the granular stress is small when $\alpha$ is small. Actually, the higher $\gamma$ is, the faster the granular stress tends to zero when $\alpha$ tends to zero.

In this model, unlike in [7], the solid volume fraction $\alpha$ can approach one. This is due to the fact that the compressibility of the solid phase is taken into account. Of course, if this solid phase is slightly compressible a volume fraction $\alpha_{2} \simeq 1$ would imply very high pressures. Mathematically this situation is not a problem, but physically it can be questioned. It is also related with the choice (1.6) for the interface pressure and velocity. This choice is physically recommended for dilute flows. When the flow is not dilute, the model, while still mathematically robust, should probably be improved.

Of course, it would be also possible to consider the most general case where the granular stress also depends on the internal energy

$$
\Theta=\Theta(\alpha, \tau, e) .
$$

However, we will see that the choice (3.11) is very interesting for the modelling and the numerics because it ensures that the volume fraction stays within its natural bounds during the pressure equilibrium resolution.

Is also possible to compute the whole thermodynamic underlying model. The expressions for the associated entropy and temperature are given in Section B. 


\subsection{Summary of the full model}

Before presenting the numerical part of our work, we sum up the model that will be used for the numerics. From now on, we will suppose that the pressure laws of the two phases are stiffened gas equations of state. We will also suppose that the granular stress is given by formula (3.11). These hypothesis are made because they permit a good balance between simplicity and generality. Of course our approach can be generalized to other physical laws. We solve

$$
W_{t}+F(W)_{x}+A(W) L(W)_{x}=\Sigma(W) .
$$

The unknowns are (with $m_{k}=\alpha_{k} \rho_{k}, E_{k}=e_{k}+u_{k}^{2} / 2, k=1,2$ )

$$
W=\left(m_{1}, m_{1} u_{1}, m_{1} E_{1}, m_{2}, m_{2} u_{2}, m_{2} E_{2}, \alpha_{1}\right)^{T} .
$$

The "conservative" flux is given by

$$
F(W)=\left(m_{1} u_{1}, m_{1} u_{1}^{2}, m_{1} E_{1} u_{1}, m_{2} u_{2}, m_{2} u_{2}^{2}, m_{2} E_{2} u_{2}, 0\right)^{T},
$$

and the "non-conservative" terms are given by

$$
\begin{aligned}
& L(W)=\left(p_{1}, p_{1} u_{1}, p_{2}, p_{2} u_{2}, \alpha_{1}\right)^{T} \\
& \begin{aligned}
A(W) L(W)_{x}= & \left(0, \alpha_{1} p_{1, x}, \alpha_{1}\left(p_{1} u_{1}\right)_{x}+p_{1}\left(u_{1}-u_{2}\right) \alpha_{1, x}, 0, \alpha_{2} p_{2, x}\right. \\
& \left.+\left(p_{2}-p_{1}\right) \alpha_{2, x}, \alpha_{2}\left(p_{2} u_{2}\right)_{x}+u_{2}\left(p_{2}-p_{1}\right) \alpha_{2, x}, v_{2} \alpha_{1, x}\right)^{T} .
\end{aligned}
\end{aligned}
$$

The pressures obey stiffened gas equations of state

$$
p_{k}=\left(\gamma_{k}-1\right) \rho_{k} e_{k}-\gamma_{k} \pi_{k}, \quad \gamma_{k}>1, \quad k=1,2 .
$$

The constants $\gamma_{k}$ and $\pi_{k}$ for $k=1,2$ are fixed and obtained from physical measurements. The source terms vector is

$$
\Sigma(W)=\left(0,0,-p_{1} P(W), 0,0, p_{1} P(W), P(W)\right)^{T} .
$$

The pressure relaxation source term is

$$
\begin{aligned}
& P(W)=\frac{1}{\tau_{P}} \alpha_{1} \alpha_{2}\left(p_{1}+R\left(\rho_{2}, \alpha_{2}\right)-p_{2}\right), \quad \tau_{P}>0, \\
& R\left(\rho_{2}, \alpha_{2}\right)=\kappa\left(\alpha_{2} \rho_{2}\right)^{\gamma_{2}}, \quad \kappa>0 .
\end{aligned}
$$

The constants $\kappa$ and $\tau_{P}$ are obtained by physical measurements. In Section 6.1, we will also present a realistic gun simulation. In this case we will propose a different expression of the source terms $\Sigma(W)$.

\section{Finite volume approach}

For the numerical implementation, we consider a finite volume discretisation and a splitting approach. The convection step is solved by a standard Rusanov scheme [16] already described in many works as [15]. In the second stage, the source terms are applied. We concentrate on the pressure relaxation source term in the next section.

For the finite volume scheme, we consider a space step $h$, a time step $\Delta t$. The cells are intervals $] x_{i-1 / 2}, x_{i+1 / 2}[$. For simplicity, we consider a regular mesh $x_{i}=i h$ (but this of course is not mandatory). The vector $W$ is approximated in each cell at time $t_{n}$ by

$$
W_{i}^{n} \simeq W\left(x_{i}, t_{n}\right)
$$


The numerical scheme for the convective terms is a standard Rusanov scheme for non-conservative systems, which reads

$$
h\left(W_{i}^{n+1,-}-W_{i}^{n}\right)+\Delta t\left(F_{i+1 / 2}^{n}-F_{i-1 / 2}^{n}\right)+\Delta t A\left(W_{i}^{n}\right) \frac{L_{i+1}^{n}-L_{i-1}^{n}}{2}=0 .
$$

The conservative numerical flux is given by

$$
\begin{aligned}
& F\left(W_{L}, W_{R}\right)=\frac{F\left(W_{L}\right)+F\left(W_{R}\right)}{2}-\zeta \frac{W_{R}-W_{L}}{2}, \\
& \zeta=\max \left(\rho\left(B\left(Y_{L}\right)\right), \rho\left(B\left(Y_{R}\right)\right)\right)
\end{aligned}
$$

where $\rho(B)$ denotes the spectral radius of the matrix $B$. The convection matrix $B(Y)$ in the primitive variables $Y$ (see (A.1)) is given in (A.3) in Section A. Our choice for the numerical viscosity parameter $\zeta$ is classical (see for example [10]). It usually leads to an entropy dissipative scheme. In exceptional cases, it may be necessary to compute the numerical viscosity from interface values instead of the cell values $Y_{L}$ and $Y_{R}$.

Our particular choice of the non-conservative terms $L$ ensures that constant velocity-pressure states will be maintained by the Rusanov scheme. Of course, in many interesting computations the velocity and pressure are not constant. However, it has been observed that one obtains better numerical results if the scheme is able to capture exactly the constant velocity and pressure solutions.

This convection step permits to obtain a value $W_{i}^{n+1,-}$ in each cell $i$. It has now to be updated in order to take into account the pressure equilibrium source and obtain $W_{i}^{n+1}$.

\section{Relaxation algorithm}

In this section, we address now the numerical approximation of the pressure relaxation source term of the system (3.13)-(3.19). As usual, we use a fractional step method in order to separate the convection step and the pressure equilibrium step.

Thus, we concentrate only on the description of the pressure equilibrium step, which can be formally written

$$
\begin{aligned}
& \alpha_{k, t}=\sigma_{k} P, \\
& m_{k, t}=u_{k, t}=0, \\
& \left(m_{k} e_{k}\right)_{t}+p_{1} \alpha_{k, t}=0 .
\end{aligned}
$$

In order to simplify the notations, we denote now by a 0 superscript the physical values in a given cell $i$ at the end of the advection step. These values are computed from the vector $W_{i}^{n+1,-}$, at the end of the convection step, given by the Rusanov scheme (4.2). The updated values at time $n+1$ are noted without any superscript.

Owing to mass and momentum conservation we have $m_{k}=m_{k}^{0}$ and $u_{k}=u_{k}^{0}$. We have now to compute $\left(\alpha_{1}, p_{1}, p_{2}\right)$ in order to pursue the computation. The system is

$$
\begin{aligned}
& p_{2}-R-p_{1}=\tau_{p} \alpha_{2, t} \\
& m_{1} e_{1}+m_{2} e_{2}=m_{1}^{0} e_{1}^{0}+m_{2}^{0} e_{2}^{0} \\
& \left(m_{1} e_{1}-m_{1}^{0} e_{1}^{0}\right)+p_{1}\left(\alpha_{1}-\alpha_{1}^{0}\right)=0 .
\end{aligned}
$$

We recall that the pressures of the two phases obey stiffened gas equations of state

$$
p_{k}\left(\rho_{k}, e_{k}\right)=\left(\gamma_{k}-1\right) \rho_{k} e_{k}-\gamma_{k} \pi_{k}, \quad \gamma_{k}>1, \quad k=1,2
$$

It is physically reasonable to suppose that

$$
\pi_{2}>\pi_{1}
$$


because phase (2) is the solid phase and because in the gas phase (1) $\pi_{1} \simeq 0$. We also recall that the granular stress $R$ is given by (3.11). Because of the stiffened gas law, we have

$$
m_{k} e_{k}=\alpha_{k} \frac{p_{k}+\gamma_{k} \pi_{k}}{\gamma_{k}-1} .
$$

Thus we have to solve for $\left(\alpha_{1}, p_{1}, p_{2}\right)$ the following three-equation system, at each time step and in each cell

$$
\begin{aligned}
& p_{2}-\kappa \alpha_{2}^{\gamma_{2}} \rho_{2}^{\gamma_{2}}-p_{1}=\frac{\tau_{p}}{\alpha_{2}\left(1-\alpha_{2}\right)} \alpha_{2, t}, \\
& \alpha_{2} \frac{p_{2}+\pi_{2}}{\gamma_{2}-1}-\alpha_{2}^{0} \frac{p_{2}^{0}+\pi_{2}}{\gamma_{2}-1}+\left(p_{1}+\pi_{2}\right)\left(\alpha_{2}-\alpha_{2}^{0}\right)=0, \\
& \alpha_{1} \frac{p_{1}+\pi_{1}}{\gamma_{1}-1}-\alpha_{1}^{0} \frac{p_{1}^{0}+\pi_{1}}{\gamma_{1}-1}+\left(p_{1}+\pi_{1}\right)\left(\alpha_{1}-\alpha_{1}^{0}\right)=0 .
\end{aligned}
$$

We have

$$
\begin{aligned}
& p_{2}-\kappa \alpha_{2}^{\gamma_{2}} \rho_{2}^{\gamma_{2}}-p_{1}=\frac{\tau_{p}}{\alpha_{2}\left(1-\alpha_{2}\right)} \alpha_{2, t}, \\
& \left(\alpha_{2}+\left(\gamma_{2}-1\right)\left(\alpha_{2}-\alpha_{2}^{0}\right)\right)\left(p_{2}+\pi_{2}\right)-\alpha_{2}^{0}\left(p_{2}^{0}+\pi_{2}\right)-\left(\gamma_{2}-1\right)\left(\alpha_{2} \rho_{2}^{\gamma_{2}} \theta\left(\alpha_{2}\right)+\tau \alpha_{2, t}\right)\left(\alpha_{2}-\alpha_{2}^{0}\right)=0, \\
& \left(\alpha_{1}+\left(\gamma_{1}-1\right)\left(\alpha_{1}-\alpha_{1}^{0}\right)\right)\left(p_{1}+\pi_{1}\right)-\alpha_{1}^{0}\left(p_{1}^{0}+\pi_{1}\right)=0 .
\end{aligned}
$$

We then note

$$
\begin{aligned}
& A_{1}=\alpha_{1}^{0}\left(p_{1}^{0}+\pi_{1}\right), \\
& A_{2}=\alpha_{2}^{0}\left(p_{2}^{0}+\pi_{2}\right) .
\end{aligned}
$$

For a stiffened gas law, the sound speed $c$ is given by the formula

$$
c=\sqrt{\frac{\gamma(p+\pi)}{\rho}} .
$$

It implies that the two quantities $A_{1}$ and $A_{2}$ are $>0$. Of course, we suppose that the initial volume fraction $0<\alpha_{2}^{0}<1$.

After the elimination of $p_{1}$ and $p_{2}$, the system can be rewritten

$$
\begin{gathered}
G\left(\alpha_{2}\right)=\left(\pi_{2}-\pi_{1}\right)\left(\alpha_{1}+\left(\gamma_{1}-1\right)\left(\alpha_{1}-\alpha_{1}^{0}\right)\right)\left(\alpha_{2}+\left(\gamma_{2}-1\right)\left(\alpha_{2}-\alpha_{2}^{0}\right)\right) \\
+\left(\kappa \alpha_{2} m_{2}^{\gamma_{2}}+\frac{\tau_{p}}{\left(1-\alpha_{2}\right)} \alpha_{2, t}-A_{2}\right)\left(\alpha_{1}+\left(\gamma_{1}-1\right)\left(\alpha_{1}-\alpha_{1}^{0}\right)\right) \\
+A_{1}\left(\alpha_{2}+\left(\gamma_{2}-1\right)\left(\alpha_{2}-\alpha_{2}^{0}\right)\right)=0 .
\end{gathered}
$$

We use an implicit first-order time discretisation of $\alpha_{2, t}$

$$
\alpha_{2, t}=\frac{\alpha_{2}-\alpha_{2}^{0}}{\Delta t}
$$

(recall that $\Delta t$ is the time step in the convection step (4.2)). The implicit approach is natural because $\tau_{p}$ may be arbitrary small and thus the source term (2.12) may be stiff. 
We first compute $G$ at the left point of the interval $[0,1]$

$$
\begin{aligned}
G(0)= & -\left(\pi_{2}-\pi_{1}\right)\left(\gamma_{2}-1\right) \alpha_{2}^{0}\left(1+\left(\gamma_{1}-1\right)\left(1-\alpha_{1}^{0}\right)\right) \\
& -\left(A_{2}+\frac{\tau_{p} \alpha_{2}^{0}}{\Delta t}\right)\left(1+\left(\gamma_{1}-1\right)\left(1-\alpha_{1}^{0}\right)\right) \\
& -A_{1}\left(\gamma_{2}-1\right) \alpha_{2}^{0}<0,
\end{aligned}
$$

because of the hypothesis (5.20) and $A_{1}, A_{2}>0$. For the computation at the right point, we introduce

$$
\beta_{1}=\frac{\gamma_{1}-1}{\gamma_{1}} \alpha_{1}^{0}
$$

We have

$$
\alpha_{1}+\left(\gamma_{1}-1\right)\left(\alpha_{1}-\alpha_{1}^{0}\right)>0 \Leftrightarrow \alpha_{1}>\beta_{1} \Leftrightarrow \alpha_{2}<1-\beta_{1} .
$$

We compute

$$
\begin{aligned}
& G\left(1-\beta_{1}\right)=A_{1}\left(1-\beta_{1}+\left(\gamma_{2}-1\right)\left(\alpha_{1}^{0}-\beta_{1}\right)\right) \\
& =\frac{A_{1}}{\gamma_{1}-1}\left(\left(\gamma_{1}-1\right)\left(1-\beta_{1}\right)+\left(\gamma_{2}-1\right) \beta_{1}\right)>0 .
\end{aligned}
$$

Thus we have existence of a solution $\alpha_{2}$ to $G\left(\alpha_{2}\right)=0$ in the interval $] 0,1-\beta_{1}[$.

We have to check that this solution leads to physically relevant pressures $p_{1}$ and $p_{2}$ i.e. that $p_{k}+\pi_{k}>0$. We have

$$
p_{1}+\pi_{1}=\frac{A_{1}}{\alpha_{1}+\left(\gamma_{1}-1\right)\left(\alpha_{1}-\alpha_{1}^{0}\right)} .
$$

This quantity is $>0$ if the solution satisfies $\alpha_{2}<1-\beta_{1}$. Finally we also have

$$
p_{2}+\pi_{2}=p_{1}+\pi_{1}+\kappa m_{2}^{\gamma_{2}}+\tau_{p} \frac{\alpha_{2}-\alpha_{2}^{0}}{\tau \alpha_{2}\left(1-\alpha_{2}\right)}+\pi_{2}-\pi_{1} .
$$

When $\alpha_{2}-\alpha_{2}^{0} \geq 0$ the previous quantity is obviously $>0$. In the case $\alpha_{2}-\alpha_{2}^{0}<0$, we use the second equation of (5.6) and we find

$$
\alpha_{2} \frac{p_{2}+\pi_{2}}{\gamma_{2}-1}=\alpha_{2}^{0} \frac{p_{2}^{0}+\pi_{2}}{\gamma_{2}-1}-\left(p_{1}+\pi_{1}+\pi_{2}-\pi_{1}\right)\left(\alpha_{2}-\alpha_{2}^{0}\right)>0 .
$$

And the algorithm can continue.

Remark 5.1. Formula (5.16) shows that we have to discard any solution that is not in the interval $] 0,1-\beta_{1}[$. Lets us notice that in many cases, we can find another solution to $G\left(\alpha_{2}\right)=0$ in $] 1-\beta_{1}, 1[$.

Remark 5.2. The previous reasoning can be easily generalized to the case where the granular stress is of the form

and the function $\theta$ satisfies

$$
R\left(\rho_{2}, \alpha_{2}\right)=\rho_{2}^{\gamma_{2}} \alpha_{2} \theta\left(\alpha_{2}\right),
$$

$$
\begin{aligned}
& \theta \text { continuous on }[0,1], \\
& \theta\left(\alpha_{2}\right) \geqslant 0, \\
& \theta\left(\alpha_{2}\right)=o\left(\alpha_{2}^{\gamma_{2}-2}\right) \text { when } \alpha_{2} \rightarrow 0 .
\end{aligned}
$$

These conditions are fulfilled by the granular stress that we proposed in (3.11) where

$$
\theta\left(\alpha_{2}\right)=\kappa \alpha_{2}^{\gamma_{2}-1}
$$


Now, we will prove that the solution $\alpha_{2}$ is unique in the interval $] 0,1-\beta_{1}[$. For this, we set

$$
f(\alpha)=\kappa \alpha+\frac{\tau_{p}}{\tau m_{2}^{\gamma_{2}}} \frac{\alpha-\alpha_{2}^{0}}{1-\alpha}
$$

it is easy to check that

$$
f \text { is convex. }
$$

We then have

$$
G^{\prime \prime}\left(\alpha_{2}\right)=-2 \gamma_{1} \gamma_{2}\left(\pi_{2}-\pi_{1}\right)-2 \gamma_{1} m_{2}^{\gamma_{2}} f^{\prime}\left(\alpha_{2}\right)+\left(\alpha_{1}+\left(\gamma_{1}-1\right)\left(\alpha_{1}-\alpha_{1}^{0}\right)\right) m_{2}^{\gamma_{2}} f^{\prime \prime}\left(\alpha_{2}\right) .
$$

The function $f$ is convex and satisfies $f(\alpha)>f(0)$ for $0<\alpha<1$. It implies that $f$ is also increasing. Then the two first terms in (5.24) are $<0$ and the last is $>0$. On the other hand, it is sufficient that $G$ is concave to prove the uniqueness. But

$$
\begin{aligned}
G^{\prime \prime}\left(\alpha_{2}\right) & \leqslant-2 \gamma_{1} \gamma_{2}\left(\pi_{2}-\pi_{1}\right)-2 \gamma_{1} m_{2}^{\gamma_{2}} f^{\prime}\left(\alpha_{2}\right)+\gamma_{1} m_{2}^{\gamma_{2}}\left(1-\alpha_{2}\right) f^{\prime \prime}\left(\alpha_{2}\right) \\
& \leqslant 2 \gamma_{1} m_{2}^{\gamma_{2}}\left[\frac{1}{2}\left(1-\alpha_{2}\right) f^{\prime \prime}\left(\alpha_{2}\right)-f^{\prime}\left(\alpha_{2}\right)-\frac{\gamma_{2}}{m_{2}^{\gamma_{2}}}\left(\pi_{2}-\pi_{1}\right)\right] .
\end{aligned}
$$

A sufficient condition to obtain uniqueness is then

$$
\frac{1}{2}\left(1-\alpha_{2}\right) f^{\prime \prime}\left(\alpha_{2}\right)-f^{\prime}\left(\alpha_{2}\right)-\frac{\gamma_{2}}{m_{2}^{\gamma_{2}}}\left(\pi_{2}-\pi_{1}\right) \leqslant 0 .
$$

The above inequality (5.26) can also be written

$$
-\kappa-\frac{\gamma_{2}}{m_{2}^{\gamma_{2}}}\left(\pi_{2}-\pi_{1}\right) \leqslant 0
$$

and it is obviously satisfied, independently of $\tau_{p}$.

We sum up the previous computations in the following proposition, which is useful for the implementation of the algorithm.

Proposition 5.1. Let the granular stress be defined by (3.11). Let

$$
\begin{aligned}
& 0<\alpha_{1}^{0}<1, \\
& p_{k}^{0}+\pi_{k}>0, \quad k=1,2,
\end{aligned}
$$

then, the algebraic system (5.2), (5.11) admits a unique solution $\left(\alpha_{1}, p_{1}, p_{2}\right)$ that complies with

$$
\begin{aligned}
& 0<\alpha_{1}<1, \\
& p_{k}+\pi_{k}>0, \quad k=1,2 .
\end{aligned}
$$

Moreover we also have

$$
\alpha_{1}>\frac{\gamma_{1}-1}{\gamma_{1}} \alpha_{1}^{0} .
$$

Finally, the solution can be computed by the Newton's method by solving equation (5.10) for $\alpha_{2}$. A safe choice for the initialization of the Newton's method is $\alpha_{2}=0$.

Proof. The proof is a consequence of the previous computations. The proposed initialization of the Newton's method comes from the concavity of $G$. 
Remark 5.3. This uniqueness result can be easily generalized to a granular stress defined by (5.19). A sufficient uniqueness condition is still (5.26) and $f$ convex but the function $f$ is now defined by

$$
f(\alpha)=\alpha^{2-\gamma_{2}} \theta(\alpha)+\frac{\tau_{p}}{\tau m_{2}^{\gamma_{2}}} \frac{\alpha-\alpha_{2}^{0}}{1-\alpha}
$$

\section{NUMERICAL RESULTS}

\subsection{Academical test cases}

First, we consider two one-dimensional test cases in order to evaluate the influence of the granular stress on the system stability. We take $\tau_{p}=0$, which corresponds to instantaneous pressure equilibrium. It is known that generally, the equilibrium system is not hyperbolic (the computations are recalled in Sect. A). The numerical parameters are taken from [2] (and also studied in [11]). We consider a simple Riemann problem in the interval $[-1 / 2,1 / 2]$. The two phases are supposed to satisfy perfect gas equations of state, with $\gamma_{1}=1.0924$ and $\gamma_{2}=1.0182$. The initial condition is made of two constant states jumping at $x=0$. We plot the solution at time $t=0.0008$. The CFL number is fixed to 0.9. The initial data are

$$
(L) \quad(R)
$$

$\begin{array}{ccc}\rho_{1} & 76.45430093 & 57.34072568 \\ u_{1} & 0 & 0 \\ p_{1} & 200 \times 10^{5} & 150 \times 10^{5} \\ \rho_{2} & 836.1239718 & 358.8982226 \\ u_{2} & 0 & 0 \\ p_{2} & 200 \times 10^{5} & 150 \times 10^{5} \\ \alpha_{1} & 0.25 & 0.25\end{array}$

We perform our algorithm with a granular stress $R=0$. With a mesh of 1000 cells we observe that the solution is rather smooth but what seems to be a small oscillation starts to develop in the center of the computational domain. The volume fraction $\alpha_{1}$, the velocities and pressures are plotted on Figures 1-3.

The same computation is made with 10000 cells. We observe on Figures 4-6 that instabilities arise, probably due to the non-hyperbolic behavior of the model.

We have also performed a computation on a 100000 cells mesh. The oscillations clearly increase as can be seen in Figure 7 for the volume fraction. Recall that thanks to the CFL condition, the Rusanov scheme computes positive cell values for void fractions and the partial masses.

We perform then another computation on the finer mesh with a granular stress given by (3.11). For the numerics, we have chosen $\kappa=500$. We observe a very slight damping of the oscillations on Figures 8 and 9 (to be carefully compared with Figs. 4 and 5). We have also plotted the two pressures on Figure 10 in order to show the difference of pressures imposed by the granular stress.

It clearly arises that the magnitude of the granular stress is not sufficient to recover a hyperbolic regime. This is confirmed in Figure 11 where we compare a $L^{2}$ norm of the imaginary parts of the eigenvalues with $R=0$ or $R>0$. The computation of the convection matrix of the equilibrium system in the case $\tau_{p} \rightarrow 0$ is given in Section A. The eigenvalues are evaluated numerically. The quantity that has been plotted is

$$
I=\sqrt{\sum_{i=1}^{6} \operatorname{Im}\left(\lambda_{i}\right)^{2}}
$$

We observe on Figure 11 that the imaginary part slightly decreases, owing to the introduction of the granular stress; however, it does not vanish.

We now evaluate the influence of the positive time scale parameter $\tau_{p}$, and on its ability to stabilize the model. While setting $p_{\text {ref }}=10^{7}$, we compare in Figure 12 the pressures obtained with different relaxation 


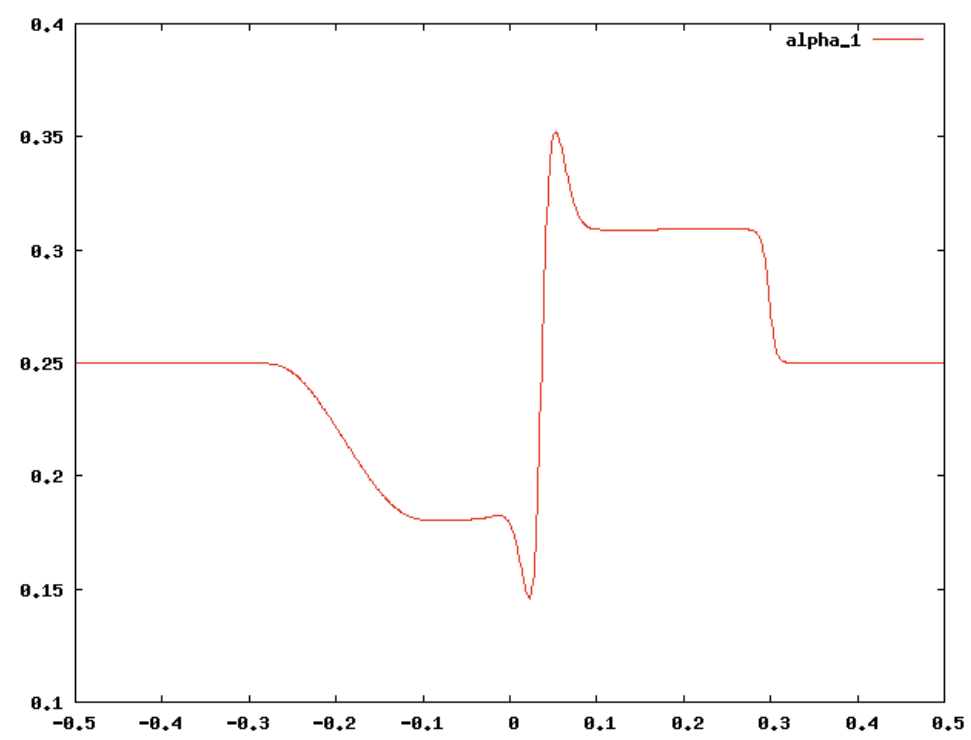

Figure 1. Void fraction, 1000 cells, $\tau_{p}=0, R=0$.

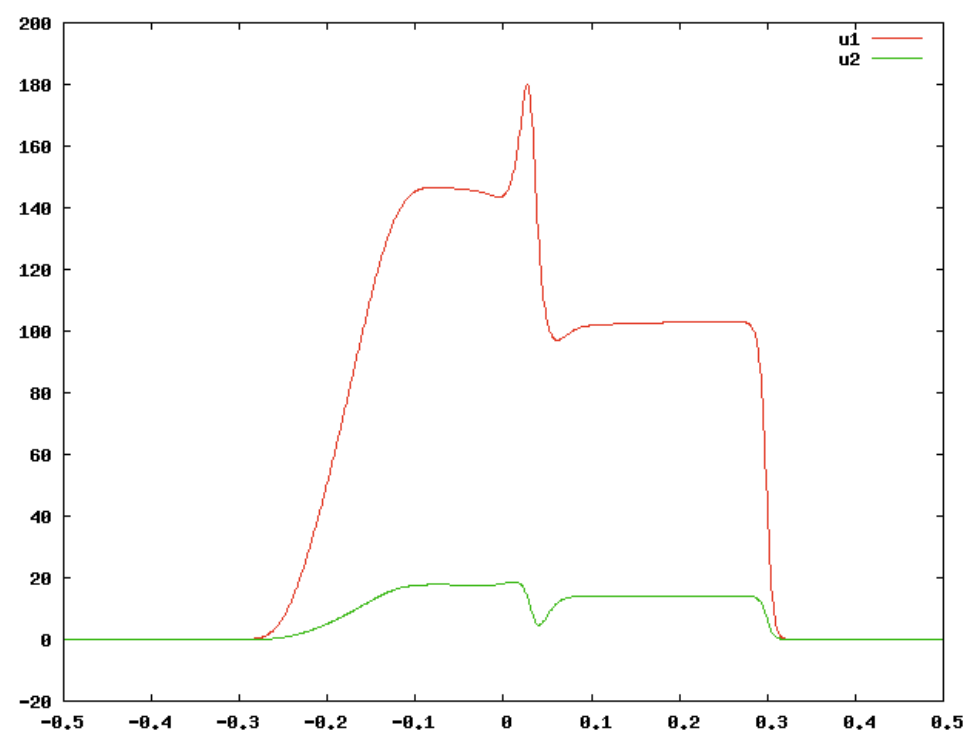

Figure 2. Velocities $u_{1}$ and $u_{2}, 1000$ cells, $\tau_{p}=0, R=0$. (Figures in color available at www.esaim-m2an.org.)

coefficients (corresponding with true time scales $\frac{\tau_{p}}{p_{\text {ref }}}=0,10^{-7}, 10^{-6}, 10^{-5}$ and $10^{-4}$ ). On this rather coarse mesh of 1000 cells, we observe that the pressures are very similar for small enough relaxation time scales. When focusing on a finer mesh of 50000 cells, the stability of the approximations increases when the relaxation time scales are larger (Fig. 13). We also observe on this test case that even with high values of $\tau_{p}$, the difference between both pressures $p_{1}$ and $p_{2}$ is indeed rather small. 


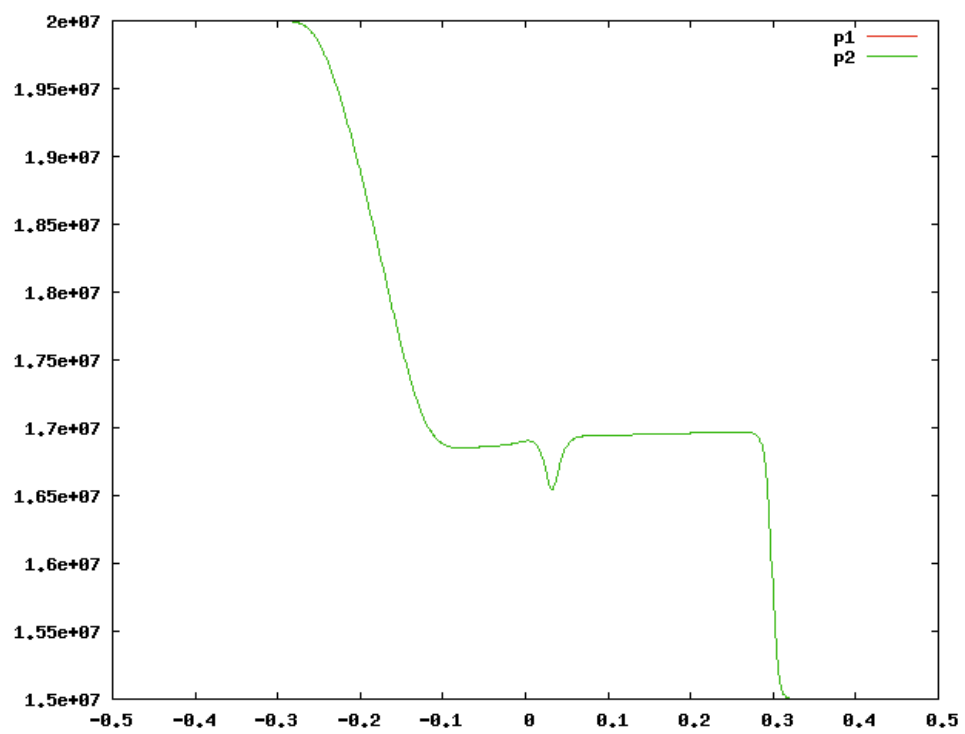

Figure 3. Pressures, 1000 cells, $\tau_{p}=0, R=0$.

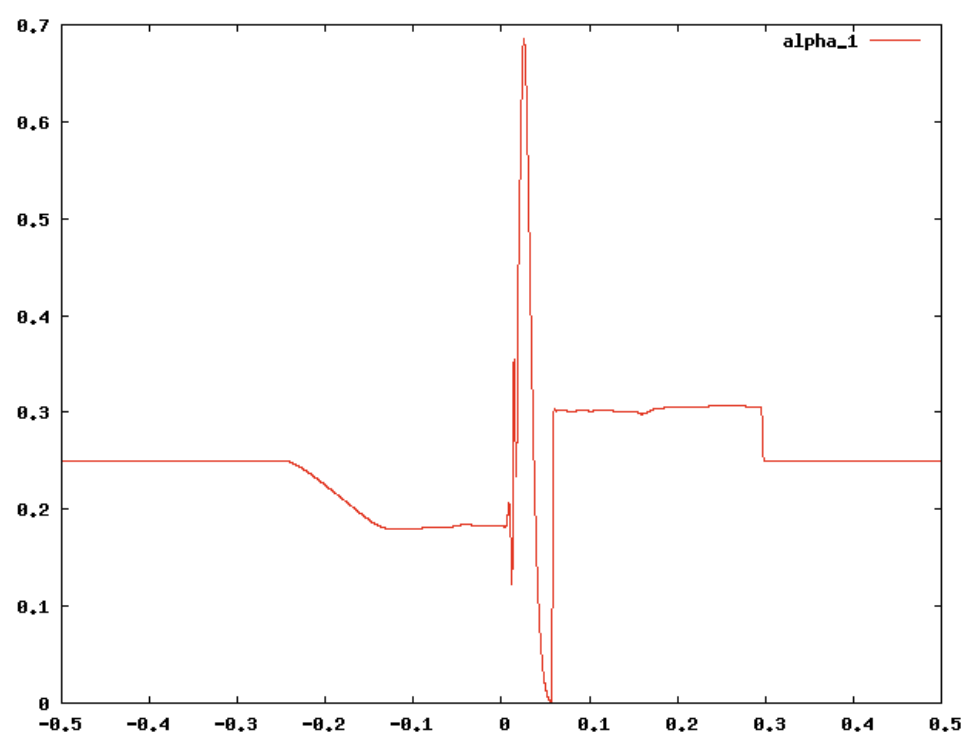

Figure 4. Void fraction, 10000 cells, $\tau_{p}=0, R=0$.

Finally, we want to compare the stabilization effect of the parameter $\tau_{P}$. For this purpose, we first set $\tau_{P}=0$, which corresponds to an instantaneous relaxation. We show in Figure 14 the evolution of the pressure plots when the mesh is refined from 10000 cells ("10k cells") to 200000 cells ("200k cells"). We observe a non-smooth behavior of the pressure graphs. It is in agreement with the fact that we approximate a non-hyperbolic, twovelocity, one-pressure model. It has also been observed in $[9,11]$. If the relaxation parameter is set to $\tau_{P}=1$, we observe on Figure 15 a stabilization of the pressure curves, which is confirmed by a grid refinement. With this relaxation parameter and with $p_{\text {ref }}=10^{7}$, the corresponding time scale given by $(2.13)$ is $t_{\text {ref }}=10^{-7}$. 


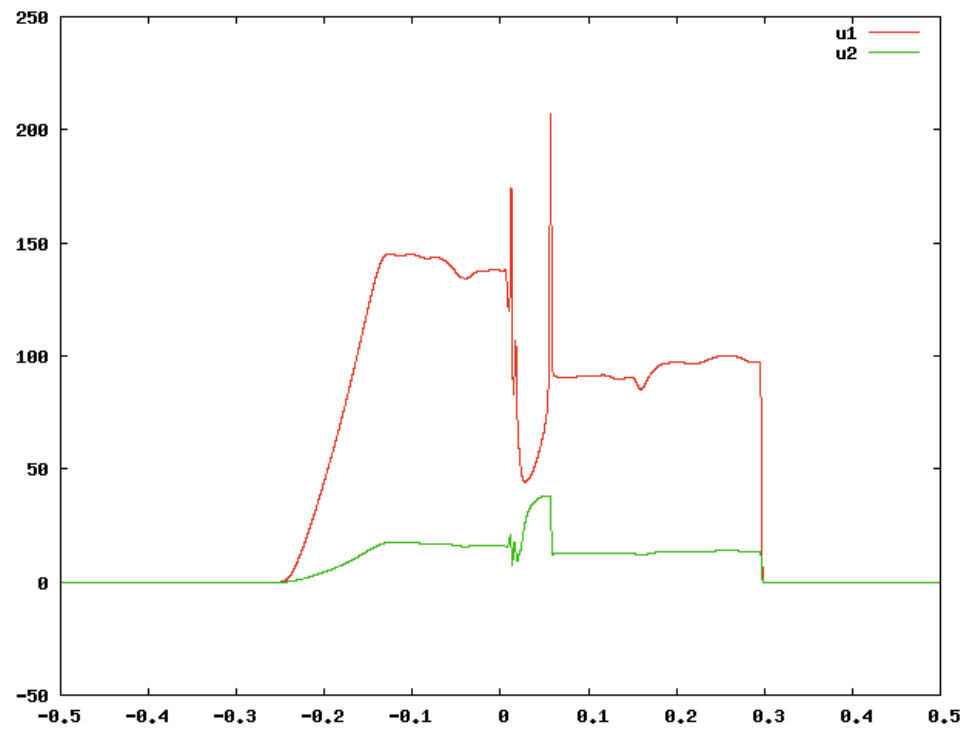

Figure 5. Velocities $u_{1}$ and $u_{2}, 10000$ cells, $\tau_{p}=0, R=0$.

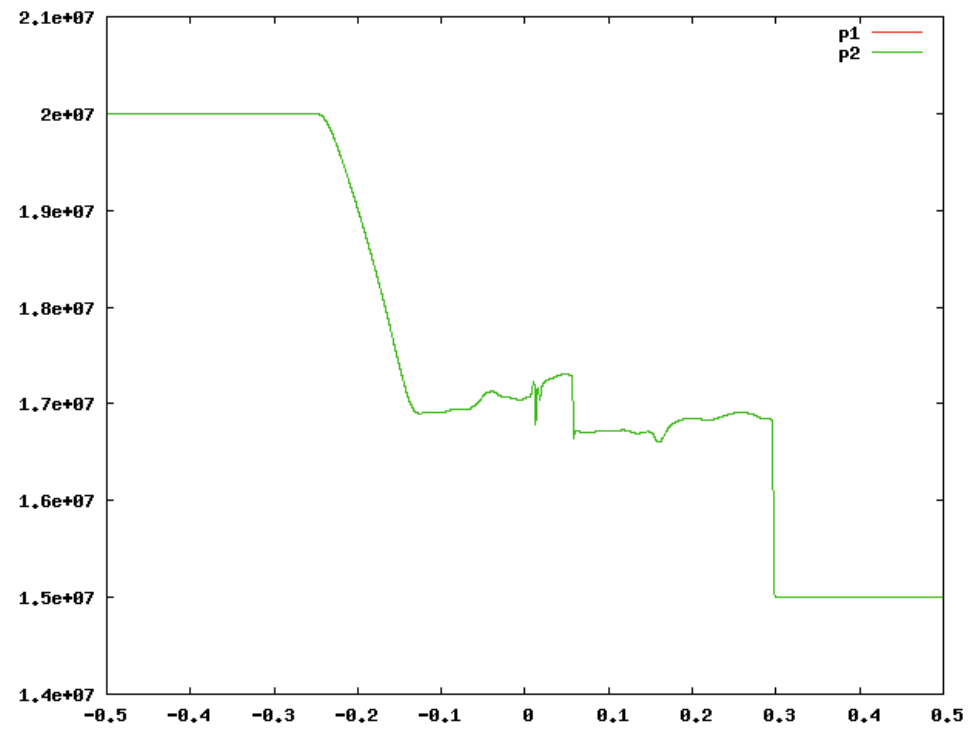

Figure 6. Pressures, 10000 cells, $\tau_{p}=0, R=0$.

In comparison, the time step $\Delta t$ for a mesh of 100000 cells is of the order of $10^{-9}$. It means that on the smaller meshes, the relaxation time scale is actually captured. It seems that the pressure is converging towards a smooth limit. It would be interesting to perform computations on even finer meshes but it would last a very long time (our longer computation lasted more than $60 \mathrm{~h}$ on eight $\mathrm{CPU}$ of a $2.0 \mathrm{GHz}$ parallel computer). 


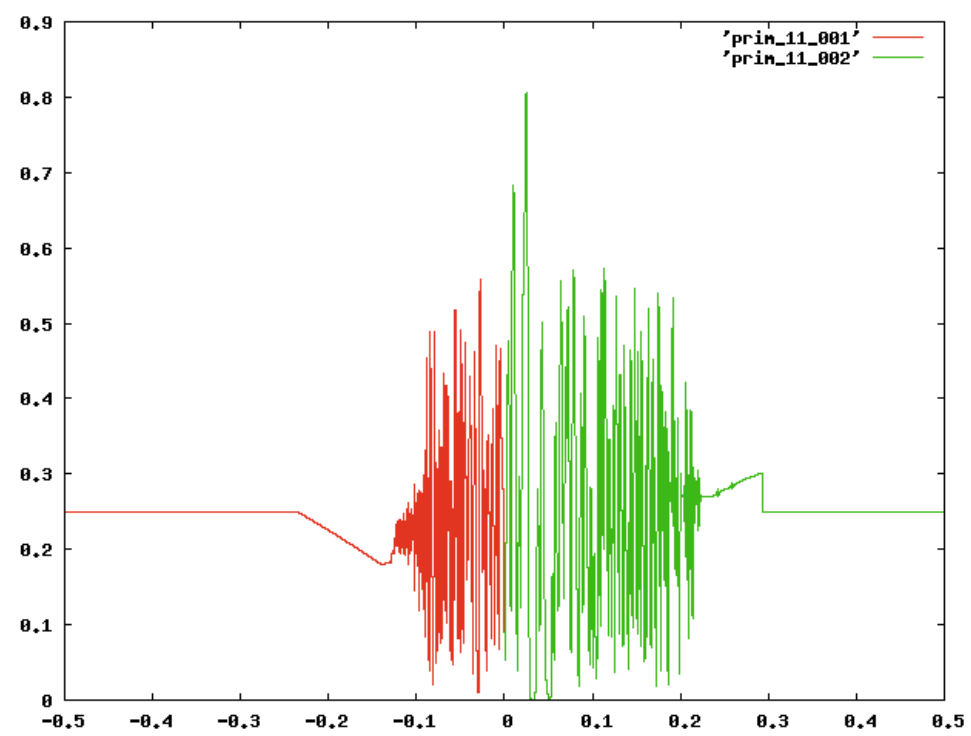

Figure 7. Void fraction, 100000 cells, $\tau_{p}=0, R=0$ (the two colors only correspond to the two processors used in the MPI computation).

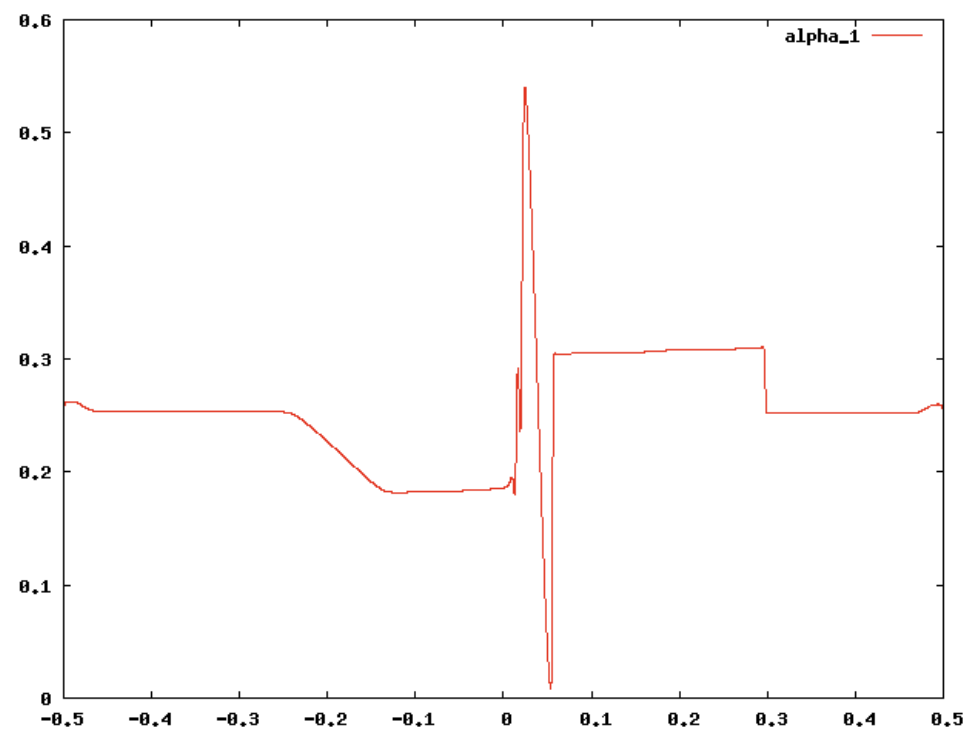

Figure 8. Void fraction, 10000 cells, $\tau_{p}=0, R=500 m_{2}^{\gamma_{2}}$.

\subsection{Simplified combustion chamber}

We consider now a more realistic case taken from [14]. We are interested in the modelling of a simplified gun. The gun is modelled by a one-dimensional tube filled with a solid phase (the powder grains) and a gas phase (the combustion gases). The breech is on the left and the shot base at the right boundary. The shot base moves according to Newton's law because the bullet is accelerated by the increase of pressure due to the combustion of the powder. For this test case, we have adapted and simplified the physical parameters given in [15]. The mass 


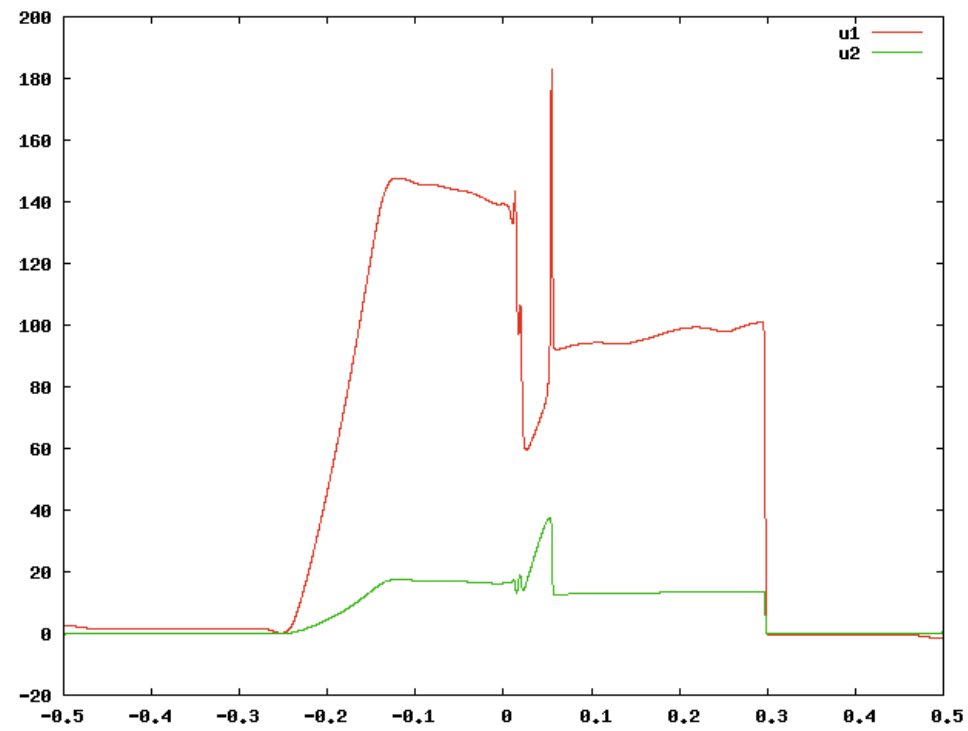

Figure 9. Velocities $u_{1}$ and $u_{2}, 10000$ cells, $\tau_{p}=0, R=500 m_{2}^{\gamma_{2}}$.

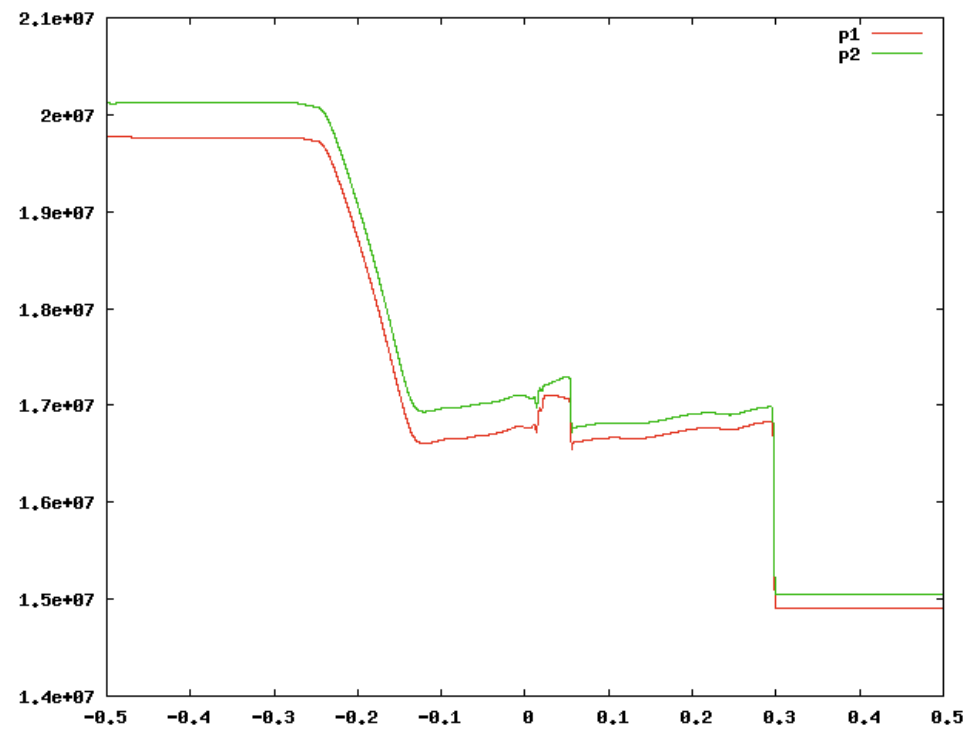

Figure 10. Pressures, 10000 cells, $\tau_{p}=0, R=500 m_{2}^{\gamma_{2}}$.

transfer term is defined by the simplified relations

$$
\begin{aligned}
& M=\alpha_{2} \rho_{2} \frac{3 \dot{r}}{r} \\
& \dot{r}=5 \times 10^{-3} \mathrm{~m} \cdot \mathrm{s}^{-1} \text { (combustion velocity of the grains) } \\
& r=10^{-3} \mathrm{~m} \text { (radius of the grains). }
\end{aligned}
$$




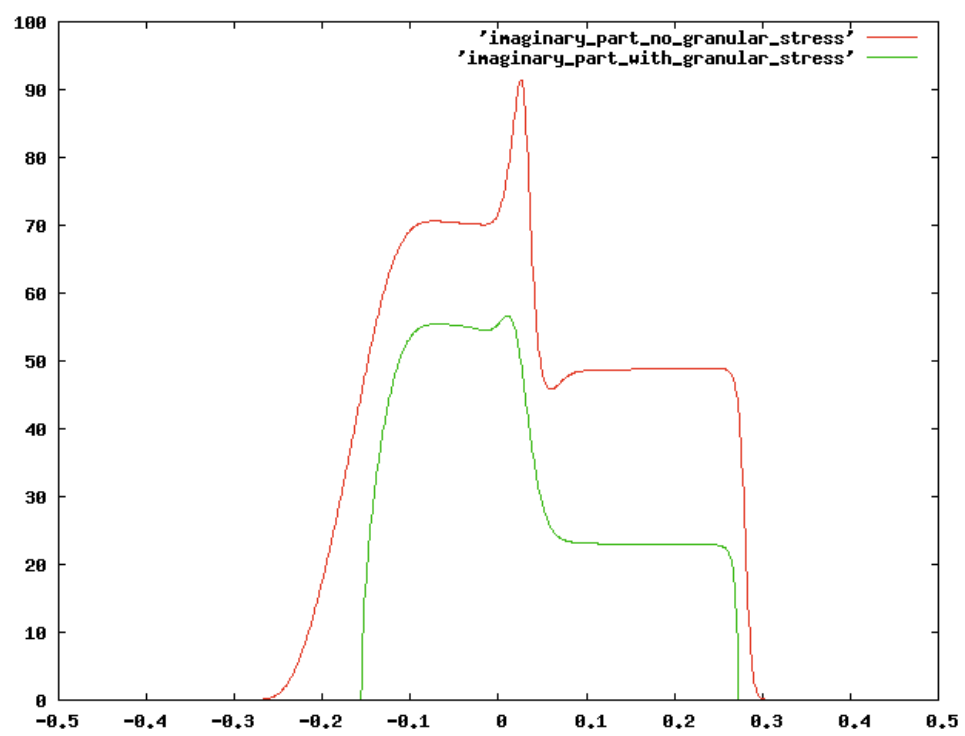

FiguRE 11. Imaginary parts, 1000 cells, $\tau_{p}=0, R=500 m_{2}^{\gamma_{2}}$ or $R=0$.

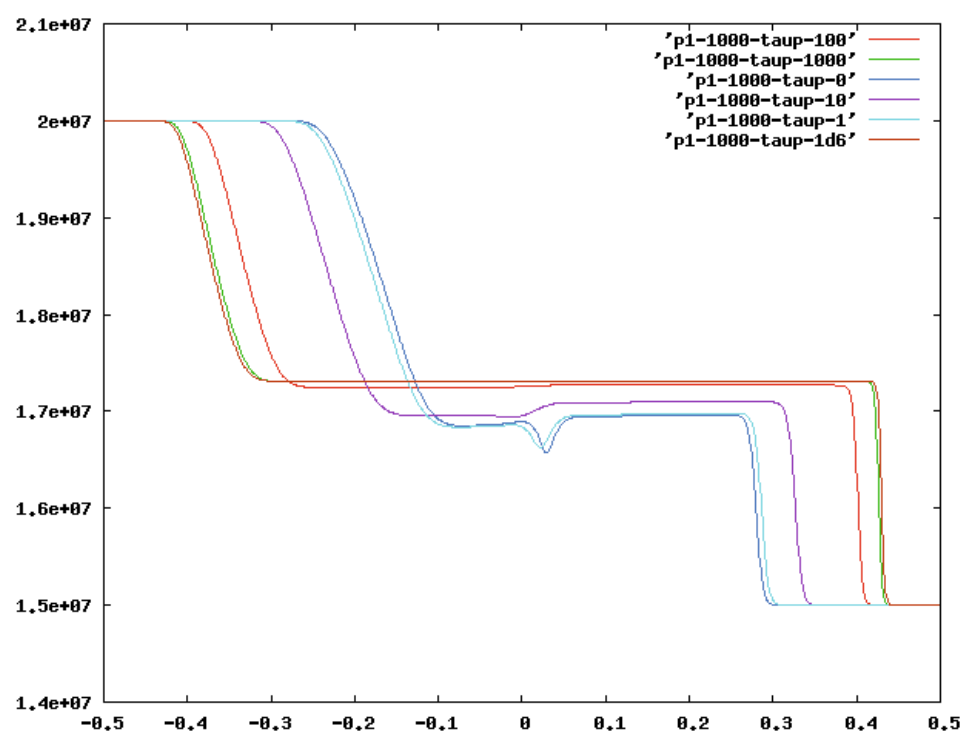

FiguRE 12. Pressure $p_{1}, 1000$ cells, with $0 \leq \tau_{p} \leq 1000$.

The momentum source term is given by

$$
\begin{aligned}
& Q=M u_{2}-D \\
& D=C \alpha_{1} \alpha_{2} \rho_{2}\left(u_{1}-u_{2}\right)\left|u_{1}-u_{2}\right| \text { (drag force) } \\
& C=\frac{3}{4 r} \text { (simplified shape factor). }
\end{aligned}
$$




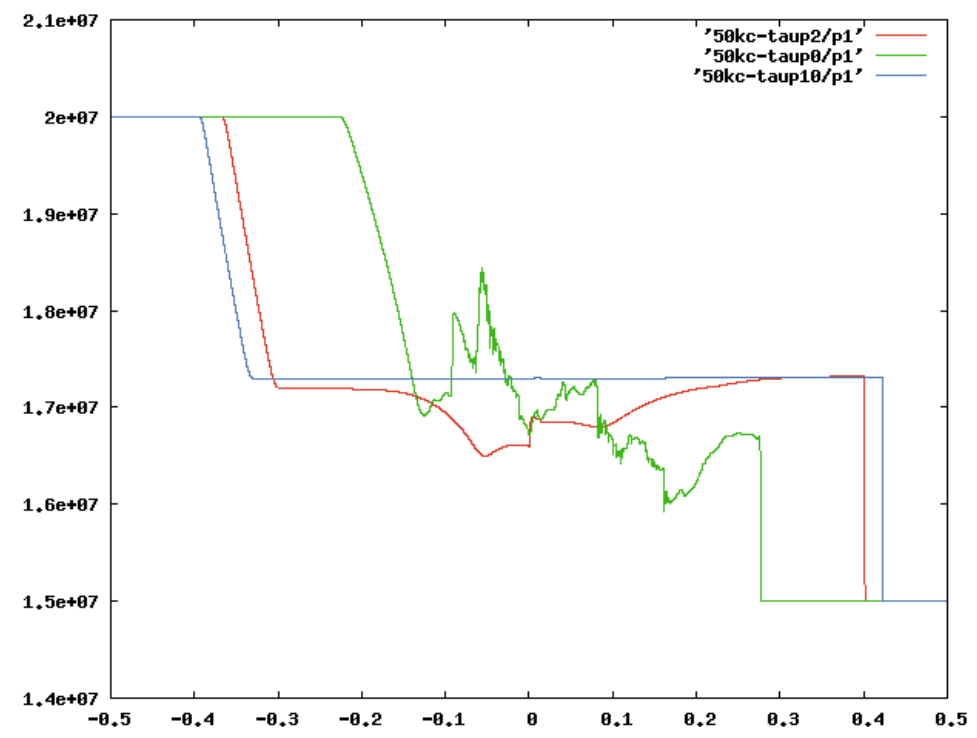

Figure 13. Pressure $p_{1}, 50000$ cells, $\tau_{p}=0,2,10, R=0$.

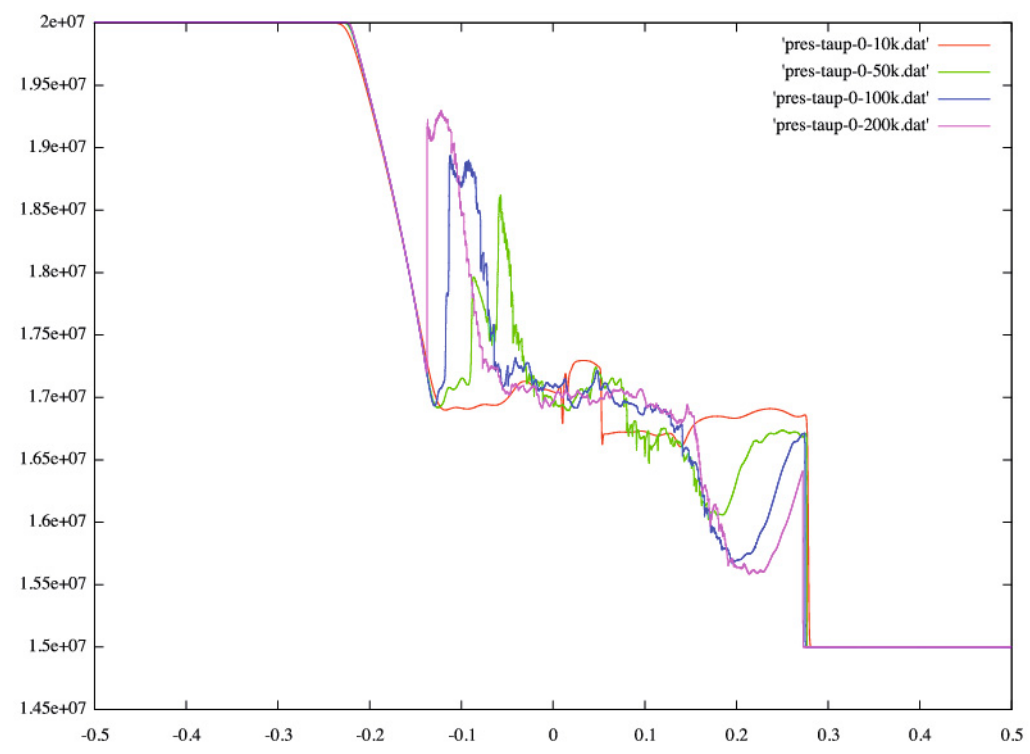

Figure 14. Pressure, 10000 to 200000 cells, $\tau_{p}=0, R=0$.

The energy source terms are

$$
\begin{aligned}
& S_{1}=-u_{2} D+M Q_{e x} \\
& S_{2}=u_{2} D \\
& Q_{e x}=37.3839 \times 10^{6} \mathrm{~J} \cdot \mathrm{kg}^{-1} \text { (chemical combustion energy). }
\end{aligned}
$$




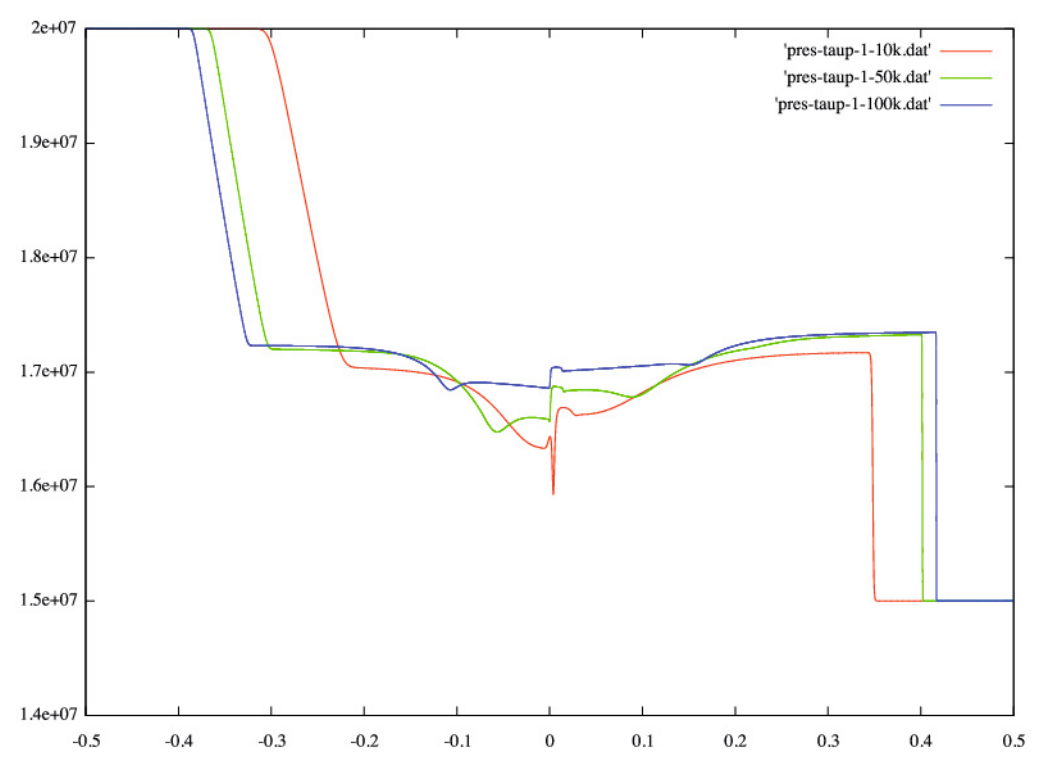

FiguRE 15. Pressure $p_{1}, 10000$ to 100000 cells, $\tau_{p}=1, R=0$.

The source term vector is now

$$
S(W)=\left(M, Q, S_{1}-p_{1} P,-M,-Q,-S_{2}+p_{1} P, P\right)^{T} .
$$

Let us remark that the energy sources do not cancel when summed up. This is only apparently a violation of the total energy conservation. Actually, we can rewrite the model in order to have opposite source terms. The rewriting is based on a translation of the internal energy in the pressure laws.

Remark 6.1. If we set

$$
e_{k}=e_{k}^{\prime}-e_{k}^{0}
$$

where $e_{k}^{\prime}$ is the translated internal energy of phase $k$, and $e_{k}^{0}$ is a reference energy for phase $k$, and if we define the translated total energies

$$
E_{k}^{\prime}=e_{k}^{\prime}+e_{k}^{0}+\frac{u_{k}^{2}}{2}
$$

The energy balance equations can be rewritten

$$
\left(m_{k} E_{k}^{\prime}\right)_{t}+\left(\left(m_{k} E_{k}^{\prime}+\alpha_{k} p_{k}\right) u_{k}\right)_{x}+p_{I} \alpha_{k, t}=\sigma_{k} S-\sigma_{k} M e_{k}^{0}
$$

Now the total translated energy $m_{1} E_{1}^{\prime}+m_{2} E_{2}^{\prime}$ is no longer conserved since the $\sigma_{k}$ terms do not cancel. The term $M\left(e_{2}^{0}-e_{1}^{0}\right)$ can be identified to the chemical reaction heat. 
The other parameters of the computations are

$$
\begin{aligned}
& \gamma_{1}=1.4 \\
& \gamma_{2}=3 \\
& \pi_{2}=2.1333 \times 10^{9} \mathrm{~Pa} \\
& \rho_{2}=1600 \mathrm{~kg} \cdot \mathrm{m}^{-3} \text { (initial solid density) } \\
& m_{p}=30 \mathrm{~kg} \text { (projectile mass) } \\
& p_{r}=10^{8} \mathrm{~Pa} \text { (resistive pressure) } \\
& p_{0}=10^{5} \mathrm{~Pa} \text { (initial pressure) } \\
& T_{0}=294 \mathrm{~K} \mathrm{(initial} \mathrm{temperature)} \\
& \rho_{0}=0.8713 \mathrm{~kg} \cdot \mathrm{m}^{-3} \text { (initial gas density) } \\
& \alpha_{2,0}=0.5709 \text { (initial solid volume fraction) } \\
& \text { diam }=132 \mathrm{~mm} \text { (diameter of the gun) } \\
& \text { length }=762 \mathrm{~mm} \text { (length of the tube) } \\
& \text { mpow }=9.5255 \mathrm{~kg} \text { (powder mass) } \\
& \kappa=0.03 \text { (granular parameter) } \\
& \text { mmol }=21.3 \mathrm{~g} \cdot \mathrm{mol}^{-1} \text { (molar mass of the gas). }
\end{aligned}
$$

The fractional step method now involves a third step following the convection step and the pressure relaxation step. It is required for integrating the remaining source terms. Because these remaining source terms are not stiff, they are integrated by a simple explicit first-order Euler method.

In addition to this third step, we also have to take into account the motion of the shot base. When the gas pressure $p_{1}$ at the shot base is greater than the resistive pressure $p_{r}$, the acceleration of the projectile is given by

$$
m_{p} \frac{\mathrm{d} v}{\mathrm{~d} t}=\left(p_{1}-p_{r}\right) \frac{\pi \mathrm{diam}^{2}}{4} .
$$

The algorithm to move the right boundary is based on an Arbitrary Lagrangian Eulerian (ALE) approach described in [14]. As the domain enlarges, the number of computational cells increases.

We compare our new compressible model with the classical Gough model described for example in [15].

We obtain the following results for the projectile velocity at the exit time

$\begin{array}{cccc} & \text { Gough model } & \text { Relax. no granular stress } & \text { Relax. with granular stress } \\ \text { velocity }\left(\mathrm{m} \cdot \mathrm{s}^{-1}\right) & 425 & 414 & 414 \\ \text { exit time }(\mathrm{ms}) & 2.9 & 3.07 & 3.07\end{array}$

In Figure 16, we compare the pressure evolution at the breech and the shot base of the projectile. We observe a good qualitative agreement between the Gough model and the relaxation model.

Finally, we plot some quantities in the tube at the final time. The porosity $\alpha_{1}$, the velocities and the pressures are given in Figures 17-19. We also plot on Figure 20 the density $\rho_{2}$ of the solid phase at the final time in order to check that the variations of the powder density are small when compared with the initial density $\rho_{2}=1600 \mathrm{~kg} \cdot \mathrm{m}^{-3}$. 


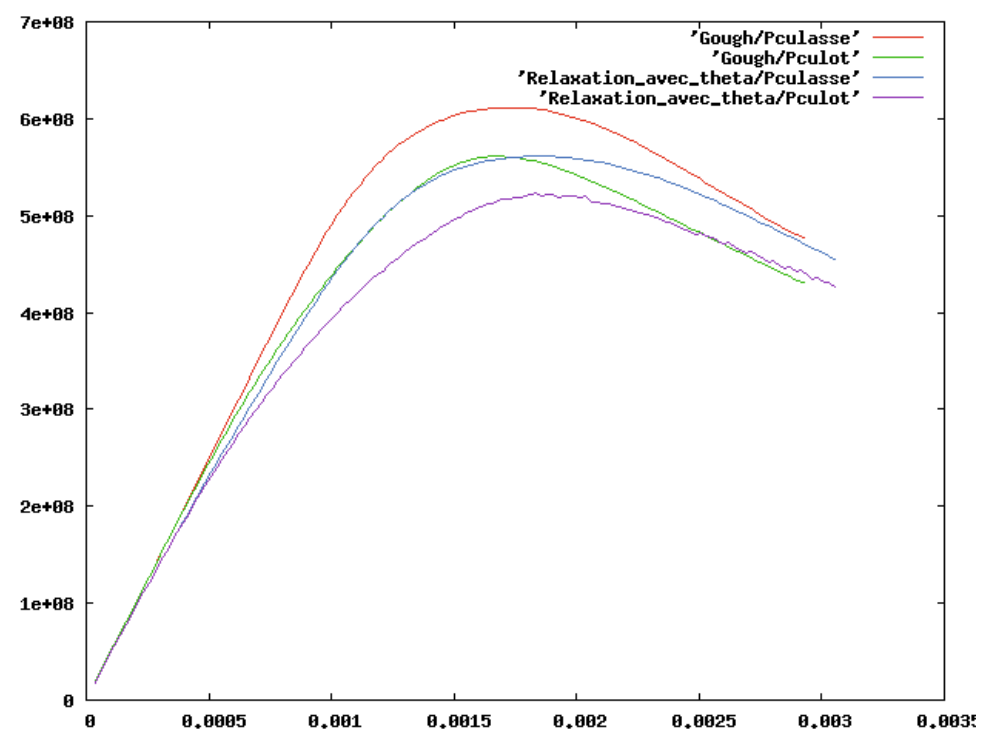

Figure 16. Pressure evolution at the breech and the shot base vs. time. Comparison between the Gough and the relaxation model.

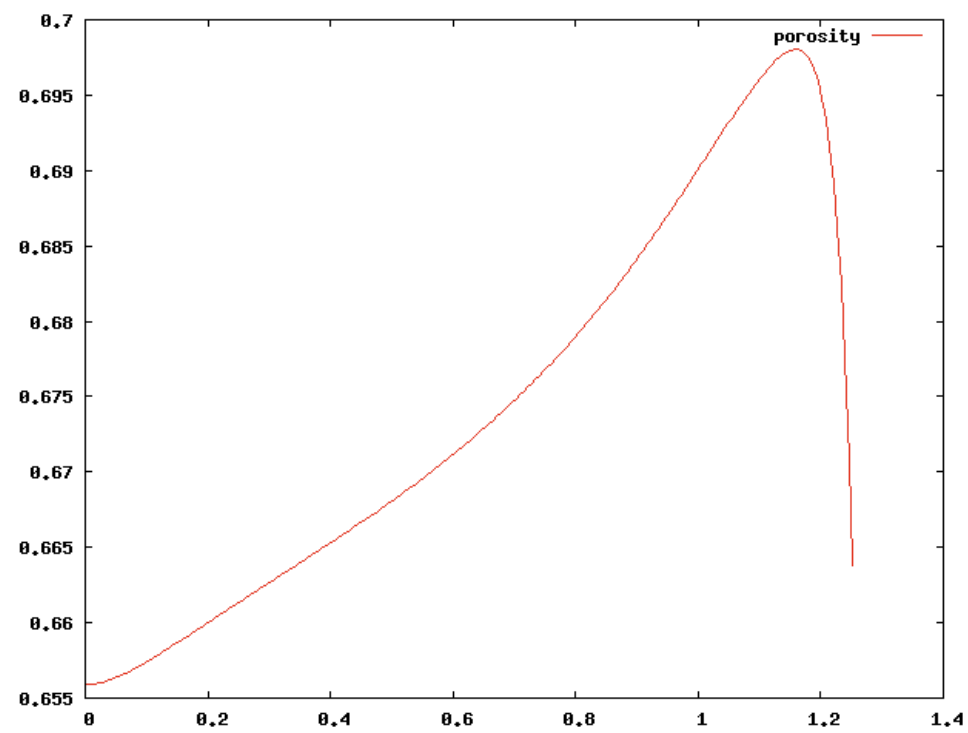

Figure 17. Porosity $\alpha_{1}$ at the final time. Relaxation model with granular stress.

\section{Conclusion}

In this paper, we have adapted the pressure relaxation method described in $[11,17]$ to the case of a nonvanishing granular stress. 


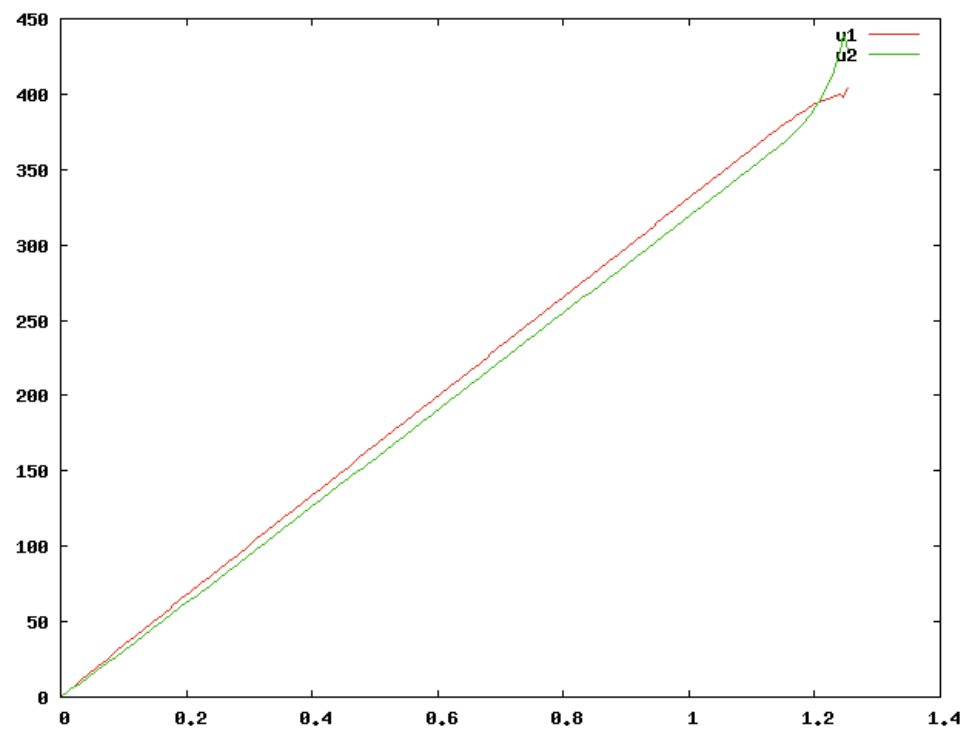

FiguRE 18. Velocities at the final time. Relaxation model with granular stress.

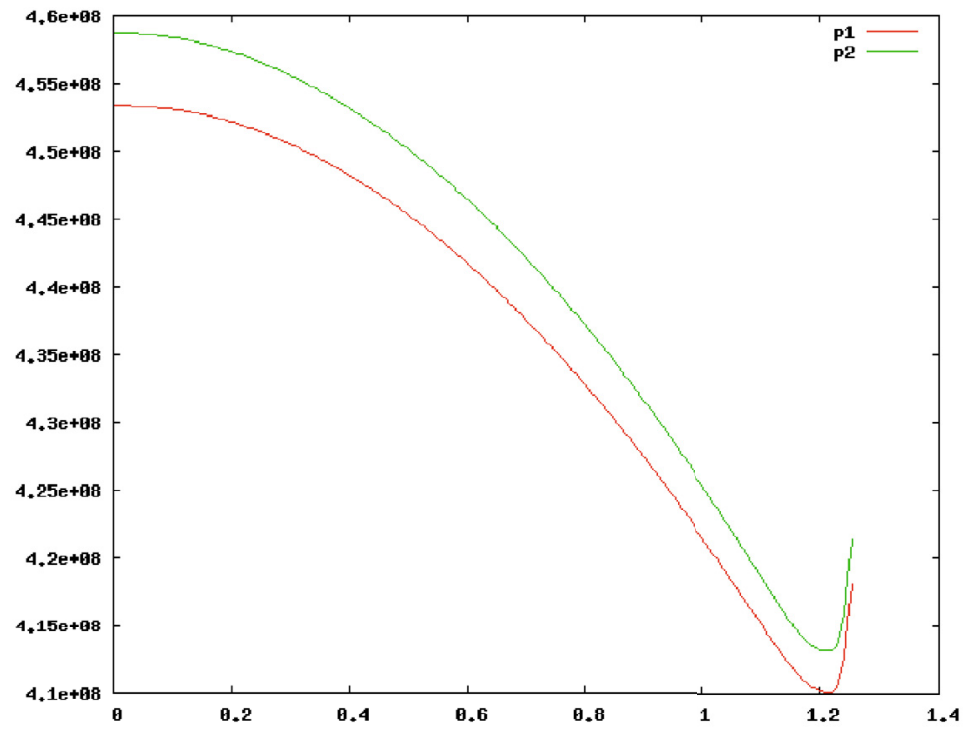

Figure 19. Pressures at the final time. Relaxation model with granular stress.

Starting from the two-velocity, two-pressure multiphase model of Baer-Nunziato, we have proposed a relaxation source term in the governing equation of the void fraction that is compatible with the second principle of thermodynamics. In this study, we have shown that

- the source term increases the entropy of the phase mixture;

- the granular stress cannot have an arbitrary form. It is related to the fact that the differential form satisfied by the entropy is closed. 


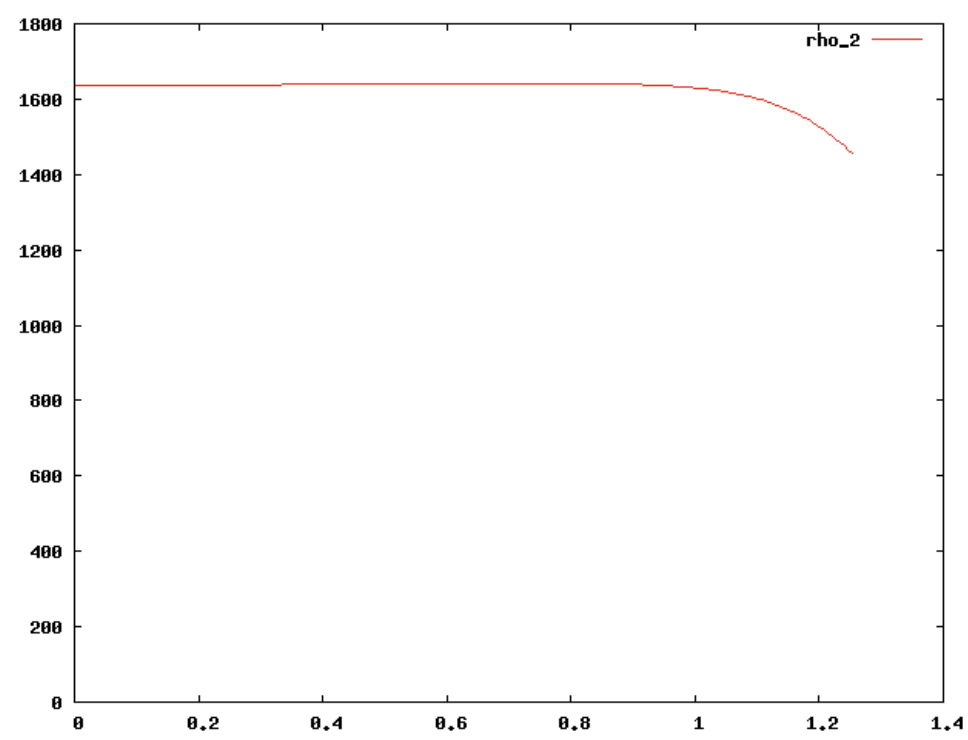

Figure 20. Density of the solid phase at the final time. Relaxation model with granular stress.

When the relaxation time tends to zero, we have then proposed a numerical method based on the underlying two-pressure model to approximate the single-pressure model. In the pressure relaxation step, the void fraction is updated in order to equilibrate the jump of pressures with the granular stress. We have proved existence and uniqueness of the equilibrium void fraction under some hypothesis on the granular stress. Those hypothesis are satisfied by physically reasonable models. In particular, it is possible to simulate in a realistic way almost incompressible solid materials. It is also possible to compute realistic granular stress with our simple granular law (3.11).

Eventually, we have proposed some numerical experiments in order to validate our approach. In an ideal test case, we have checked that when the mesh is refined, the instability of the one-pressure model is (fortunately) not suppressed. We also checked that the introduction of the granular stress slightly improves the whole stability. We finally performed more realistic simulations where we were able to reproduce correct quantitative features of a simplified gun.

The whole approach is thus very promising and must now be extended to more sophisticated granular pressure laws, equations of state and geometries.

\section{A. Appendix I: Hyperbolicity}

\section{A.1. Relaxed system}

For the sake of completeness, we recall the proof of hyperbolicity of the convection part of the equations (1.3). It is convenient to study it in the variables

$$
Y=\left(\alpha_{1}, \rho_{1}, u_{1}, s_{1}, \rho_{2}, u_{2}, s_{2}\right)^{T}
$$

In this set of variables the system becomes

$$
Y_{t}+B(Y) Y_{x}=0,
$$


with

$$
\begin{array}{ll}
c_{k}=\frac{\partial p_{k}\left(\rho_{k}, s_{k}\right)}{\partial \rho_{k}}, \quad k=1,2 \\
B(Y)=\left[\begin{array}{ccccccc}
u_{2} & & & & & & \\
\frac{\rho_{1}\left(u_{1}-u_{2}\right)}{\alpha_{1}} & u_{1} & \rho_{1} & & & & \\
& \frac{c_{1}^{2}}{\rho_{1}} & u_{1} & \frac{p_{1, s_{1}}}{\rho_{1}} & & & \\
& & & u_{1} & & & \\
& & & & u_{2} & \rho_{2} & \\
\frac{p_{1}-p_{2}}{m_{2}} & & & & \frac{c_{2}^{2}}{\rho_{2}} & u_{2} & \frac{p_{2, s_{2}}}{\rho_{2}} \\
& & & & & & u_{2}
\end{array}\right] .
\end{array}
$$

The characteristic polynomial is

$$
P(\lambda)=\left(u_{2}-\lambda\right)^{2}\left(u_{1}-\lambda\right)\left(u_{1}-c_{1}-\lambda\right)\left(u_{1}+c_{1}-\lambda\right)\left(u_{2}-c_{2}-\lambda\right)\left(u_{2}+c_{2}-\lambda\right) .
$$

We can then state the following proposition:

Proposition A.1. If $\left|u_{1}-u_{2}\right| \neq c_{k}, k=1,2$ then, the system (1.3) is hyperbolic (its eigenvalues are real and it has a full set of eigenvectors). If $\left|u_{1}-u_{2}\right|=c_{k}$ for $k=1$ or 2 then the system is resonant (its eigenvalues are real but $B(Y)$ is not diagonalizable).

\section{A.2. Equilibrium system}

We also study the hyperbolicity behavior of the equilibrium system, which correspond to the limit $\tau_{p}=0$. When the granular stress vanishes, the results are given in several papers. We thus only detail the case $R>0$ with a granular stress satisfying (3.10). The computations given below have been used to draw Figure 11. We note, for any quantity $z$,

$$
D_{k} z=z_{t}+u_{k} z_{x}
$$

At equilibrium, we can remove the transport equations in $\alpha_{k}$ and replace them by the pressure relation

$$
p_{2}=p_{1}+\alpha_{2} \rho_{2}^{\gamma_{2}} \theta\left(\alpha_{2}\right)=p_{1}+\rho_{2}^{\gamma_{2}} g\left(\alpha_{2}\right) .
$$

We note $h$ the inverse function of $g$,

$$
\forall \alpha \in] 0,1[\quad h(g(\alpha))=\alpha .
$$

Of course, when the granular stress vanishes, the inverse function of $g$ is not defined and the computations must be carried out in another way. At equilibrium, we have

$$
\begin{aligned}
& \alpha_{2}=h\left(\frac{p_{2}-p_{1}}{\rho_{2}^{\gamma_{2}}}\right) \\
& \Rightarrow \mathrm{d} \alpha_{2}=\delta\left(\left(c_{2}^{2}-\gamma_{2} \frac{p_{2}-p_{1}}{\rho_{2}}\right) \mathrm{d} \rho_{2}+p_{2, s_{2}} \mathrm{~d} s_{2}-c_{1}^{2} \mathrm{~d} \rho_{1}-p_{1, s_{1}} \mathrm{~d} s_{1}\right) \\
& \text { with } \delta=\frac{h^{\prime}\left(\frac{p_{2}-p_{1}}{\rho_{2}^{\gamma / 2}}\right)}{\rho_{2}^{\gamma_{2}}}>0 .
\end{aligned}
$$

Example A.1. We can consider

We then have

$$
\theta(\alpha)=\kappa \alpha^{\gamma_{2}-1}
$$

$$
\delta=\frac{\alpha_{2}^{1-1 / \gamma_{2}}}{\kappa \gamma_{2} \rho_{2}^{\gamma_{2}}}
$$


It is natural to introduce

In such a way that we have also

$$
a_{2}^{2}=\frac{\gamma_{2} \rho_{1}}{\gamma_{1} \rho_{2}} c_{1}^{2}+\gamma_{2} \frac{\pi_{2}-\pi_{1}}{\rho_{2}}>0
$$

$$
\mathrm{d} \alpha_{2}=\delta\left(a_{2}^{2} \mathrm{~d} \rho_{2}+p_{2, s_{2}} \mathrm{~d} s_{2}-c_{1}^{2} \mathrm{~d} \rho_{1}-p_{1, s_{1}} \mathrm{~d} s_{1}\right)
$$

It gives another expression of the source term $P$ at equilibrium

$$
P=-\delta\left(a_{2}^{2} D_{2} \rho_{2}+p_{2, s_{2}} D_{2} s_{2}-c_{1}^{2} D_{2} \rho_{1}-p_{1, s_{1}} D_{2} s_{1}\right) .
$$

We then rewrite the equilibrium system in the variables

$$
Z=\left(\rho_{1}, u_{1}, s_{1}, \rho_{2}, u_{2}, s_{2}\right)^{T}
$$

In these variables, the system is

$$
Z_{t}+C(Z) Z_{x}=0 .
$$

For the sake of completeness, we give some details of the computations

$$
\begin{aligned}
\rho_{1, t} & +u_{1} \rho_{1, x}-\frac{\rho_{1}}{\alpha_{1}}\left(u_{1}-u_{2}\right) \delta\left(a_{2}^{2} \rho_{2, x}+p_{2, s_{2}} s_{2, x}-c_{1}^{2} \rho_{1, x}-p_{1, s_{1}} s_{1, x}\right)+\rho_{1} u_{1, x} \\
& -\frac{\rho_{1}}{\alpha_{1}} \delta\left(a_{2}^{2} D_{2} \rho_{2}+p_{2, s_{2}} D_{2} s_{2}-c_{1}^{2} D_{2} \rho_{1}-p_{1, s_{1}} D_{2} s_{1}\right)=0 \\
\rho_{2, t} & +u_{2} \rho_{2, x}+\frac{\rho_{2}}{\alpha_{2}} \delta\left(a_{2}^{2} D_{2} \rho_{2}+p_{2, s_{2}} D_{2} s_{2}-c_{1}^{2} D_{2} \rho_{1}-p_{1, s_{1}} D_{2} s_{1}\right)+\rho_{2} u_{2, x}=0
\end{aligned}
$$

$$
\begin{aligned}
& \rho_{1, t}+u_{1} \rho_{1, x}-\frac{\rho_{1}}{\alpha_{1}} u_{1} \delta\left(a_{2}^{2} \rho_{2, x}+p_{2, s_{2}} s_{2, x}-c_{1}^{2} \rho_{1, x}-p_{1, s_{1}} s_{1, x}\right)+\rho_{1} u_{1, x} \\
& \quad-\frac{\rho_{1}}{\alpha_{1}} \delta\left(a_{2}^{2} \rho_{2, t}+p_{2, s_{2}} s_{2, t}-c_{1}^{2} \rho_{1, t}-p_{1, s_{1}} s_{1, t}\right)=0, \quad(\mathrm{~A} .17) \\
& u_{1, t}+u_{1} u_{1, x}+\frac{1}{\rho_{1}} p_{1, x}=0, \quad u_{2, t}+u_{2} u_{2, x}+\frac{1}{\rho_{2}} p_{2, x}+\frac{p_{2}-p_{1}}{m_{2}} \delta\left(a_{2}^{2} \rho_{2, x}+p_{2, s_{2}} s_{2, x}-c_{1}^{2} \rho_{1, x}-p_{1, s_{1}} s_{1, x}\right)=0 \\
& \left(1+\frac{\rho_{1} c_{1}^{2} \delta}{\alpha_{1}}\right) \rho_{1, t}-\frac{\rho_{1} a_{2}^{2} \delta}{\alpha_{1}} \rho_{2, t}+u_{1} \rho_{1, x}+\frac{\rho_{1}}{\alpha_{1}} \delta\left(-a_{2}^{2} u_{1} \rho_{2, x}+c_{1}^{2} u_{1} \rho_{1, x}+\left(u_{2}-u_{1}\right) p_{2, s_{2}} s_{2, x}\right)+\rho_{1} u_{1, x}=0 \\
& \left(1+\frac{\rho_{2} a_{2}^{2} \delta}{\alpha_{2}}\right) \rho_{2, t}-\frac{\rho_{2} c_{1}^{2} \delta}{\alpha_{2}} \rho_{1, t}+u_{2} \rho_{2, x}+\frac{\rho_{2}}{\alpha_{2}} \delta\left(a_{2}^{2} u_{2} \rho_{2, x}-c_{1}^{2} u_{2} \rho_{1, x}-p_{1, s_{1}}\left(u_{2}-u_{1}\right) s_{1, x}\right)+\rho_{2} u_{2, x}=0 \\
& (\mathrm{~A} .20) \\
& \left(1+\frac{\rho_{1} c_{1}^{2} \delta}{\alpha_{1}}\right) \rho_{1, t}-\frac{\rho_{1} a_{2}^{2} \delta}{\alpha_{1}} \rho_{2, t}+\left(1+\frac{\rho_{1} c_{1}^{2} \delta}{\alpha_{1}}\right) u_{1} \rho_{1, x}+\frac{\rho_{1}}{\alpha_{1}} \delta\left(-a_{2}^{2} u_{1} \rho_{2, x}+p_{2, s_{2}}\left(u_{2}-u_{1}\right) s_{2, x}\right)+\rho_{1} u_{1, x}=0 \\
& \left(1+\frac{\rho_{2} a_{2}^{2} \delta}{\alpha_{2}}\right) \rho_{2, t}-\frac{\rho_{2} c_{1}^{2} \delta}{\alpha_{2}} \rho_{1, t}+\left(1+\frac{\rho_{2} a_{2}^{2} \delta}{\alpha_{2}}\right) u_{2} \rho_{2, x}+\frac{\rho_{2}}{\alpha_{2}} \delta\left(-c_{1}^{2} u_{2} \rho_{1, x}-p_{1, s_{1}}\left(u_{2}-u_{1}\right) s_{1, x}\right)+\rho_{2} u_{2, x}=0
\end{aligned}
$$




$$
\begin{array}{r}
u_{1, t}+u_{1} u_{1, x}+\frac{c_{1}^{2}}{\rho_{1}} \rho_{1, x}+\frac{p_{1, s_{1}}}{\rho_{1}} s_{1, x}=0, \quad u_{2, t}+u_{2} u_{2, x}+\frac{1}{\rho_{2}}\left(c_{2}^{2}+\frac{p_{2}-p_{1}}{\alpha_{2}} \delta a_{2}^{2}\right) \rho_{2, x}+\frac{p_{2, s_{2}}}{\rho_{2}}\left(1+\frac{p_{2}-p_{1}}{\alpha_{2}} \delta\right) s_{2, x} \\
+\frac{p_{2}-p_{1}}{m_{2}} \delta\left(-c_{1}^{2} \rho_{1, x}-p_{1, s_{1}} s_{1, x}\right)=0 . \quad(\mathrm{A} .22)
\end{array}
$$

Finally, setting

we find

$$
\Delta=\alpha_{1} \alpha_{2}+\delta\left(\alpha_{1} \rho_{2} a_{2}^{2}+\alpha_{2} \rho_{1} c_{1}^{2}\right)
$$

$C(Z)=$

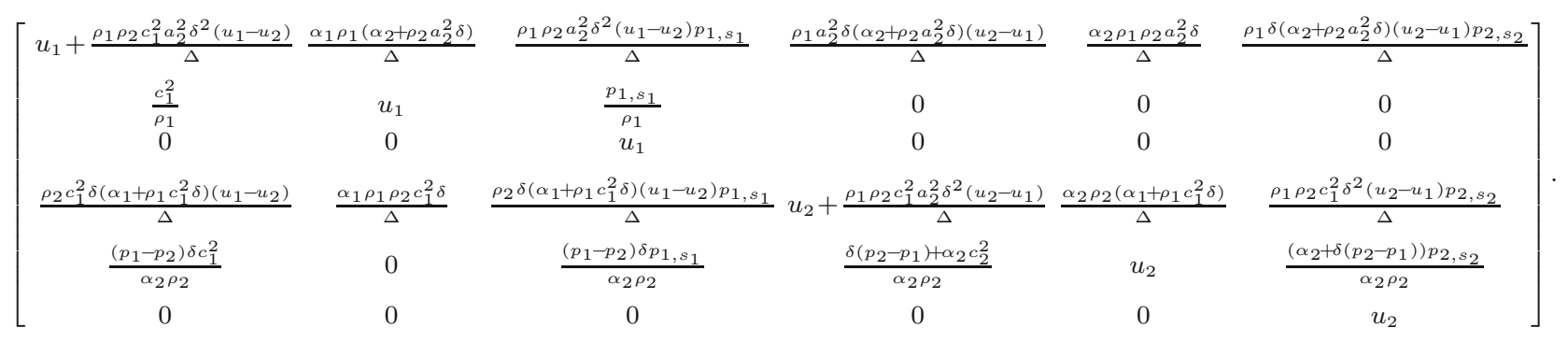

It is not easy to compute the eigenvalues analytically. It is also difficult to give a practical sufficient condition on all the parameters in order to prove that the eigenvalues are all real. In the case $\delta=0$, corresponding to an infinite granular stress, the characteristic polynomial is

$$
P(\lambda)=\left(u_{2}-\lambda\right)\left(u_{1}-\lambda\right)\left(u_{1}-c_{1}-\lambda\right)\left(u_{1}+c_{1}-\lambda\right)\left(u_{2}-c_{2}-\lambda\right)\left(u_{2}+c_{2}-\lambda\right) .
$$

We recover the same eigenvalues as in (A.4).

With a small $\kappa$, which corresponds to a big $\delta$ as can be seen by formula (A.10), we observe numerically that the system is elliptic when $u_{1} \neq u_{2}$. When $\kappa$ increases, $\delta$ decreases and we recover a hyperbolic behavior.

Numerical application. We take

$$
\begin{aligned}
& \gamma_{1}=1.0924 \\
& \gamma_{2}=1.0182 \\
& \pi_{1}=\pi_{2}=0 \\
& \alpha_{1}=0.25 \\
& p_{1}=0.2 \times 10^{8} \\
& \kappa=0.01 \Rightarrow p_{2}=0.20000007050881 \times 10^{8} \\
& u_{2}=-u_{1}=50 \\
& \rho_{1}=76.45430093 \\
& \rho_{2}=836.1239718 .
\end{aligned}
$$

The eigenvalues are

$$
\begin{aligned}
& -310.79 \\
& -50.00 \\
& 48.96-9.36 i \\
& 48.96+9.36 i \\
& 50
\end{aligned}
$$

212.86 . 
We modify $\kappa$ to $\kappa=500$, the pressure $p_{2}$ is now $p_{2}=0.20352544 \times 10^{8}$. The eigenvalues become real

$$
\begin{aligned}
& -312.54 \\
& -50.000 \\
& 30.507 \\
& 50.000 \\
& 67.438 \\
& 214.59 .
\end{aligned}
$$

\section{B. Appendix II: Associated entropy}

It is also possible to compute an entropy associated to the choice (3.11). For this, we postulate the following form of the entropy of the solid phase (as in Sect. 3, we omit the subscript)

$$
s=K(\alpha) U\left((e-\pi \tau) \tau^{\gamma-1}\right) .
$$

This choice is justified by the fact that when $K$ is constant, then we recover the general entropy of a stiffened gas. We can verify that our entropy and our stiffened gas law are compatible. Without the subscripts, the equation (2.5) reads

The temperature is given by

$$
T \mathrm{~d} s=\mathrm{d} e+p \mathrm{~d} \tau-\Theta \mathrm{d} \alpha .
$$

$$
s_{e}=\frac{1}{T}=K(\alpha) \tau^{\gamma-1} U^{\prime}\left((e-\pi \tau) \tau^{\gamma-1}\right) .
$$

In a similar way, we can deduce a relationship between $p$ and $T$

$$
s_{\tau}=\frac{p}{T}=p s_{e} .
$$

This relation enables to compute the pressure

$$
\begin{aligned}
p & =\frac{s_{\tau}}{s_{e}} \\
& =\frac{K(\alpha)\left((\gamma-1) e \tau^{\gamma-2}-\gamma \pi \tau^{\gamma-1}\right) U^{\prime}\left((e-\pi \tau) \tau^{\gamma-1}\right)}{K(\alpha) \tau^{\gamma-1} U^{\prime}\left((e-\pi \tau) \tau^{\gamma-1}\right)} \\
& =(\gamma-1) \frac{e}{\tau}-\gamma \pi
\end{aligned}
$$

and we indeed recover the stiffened gas equation of state.

We try now to find an expression for the function $U(x)$. From (B.2) we can write

$$
s_{\alpha}=-\frac{\Theta}{T}
$$

and thus

$$
\Theta=-\frac{s_{\alpha}}{s_{e}}=-\frac{K^{\prime}(\alpha)}{K(\alpha)} \frac{U\left((e-\pi \tau) \tau^{\gamma-1}\right)}{U^{\prime}\left((e-\pi \tau) \tau^{\gamma-1}\right)} \rho^{\gamma-1} .
$$

But $\Theta$ has also to be of the form (3.10). It implies that

$$
\begin{aligned}
& K(\alpha)=B \exp \left(\int_{0}^{\alpha} \theta(u) \mathrm{d} u\right), \\
& U(x)=-A \exp (-B x) .
\end{aligned}
$$


We choose now the sign of the constants $A$ and $B$ in such way that the temperature is positive and that the function $U$ is concave. It implies that $A$ and $B$ are $>0$. The positivity of $T$ and the concavity of $U$ are required for obtaining real sound speeds in the pure phases (see [6]).

\section{REFERENCES}

[1] M.R. Baer and J.W. Nunziato, A two phase mixture theory for the deflagration to detonation transition (ddt) in reactive granular materials. Int. J. Multiph. Flow 16 (1986) 861-889.

[2] F. Coquel, K. El Amine, E. Godlewski, B. Perthame and P. Rascle, A numerical method using upwind schemes for the resolution of two-phase flows. J. Comput. Phys. 136 (1997) 272-288.

[3] F. Coquel, T. Gallouët, J.-M. Hérard and N. Seguin, Closure laws for a two-fluid two-pressure model. C. R. Math. Acad. Sci. Paris 334 (2002) 927-932.

[4] T. Gallouët, J.-M. Hérard and N. Seguin, Numerical modeling of two-phase flows using the two-fluid two-pressure approach. Math. Models Methods Appl. Sci. 14 (2004) 663-700.

[5] D. Gidaspow, Multiphase flow and fluidization - Continuum and kinetic theory descriptions. Academic Press Inc., Boston, USA (1994).

[6] E. Godlewski and P.-A. Raviart, Numerical approximation of hyperbolic systems of conservation laws, Applied Mathematical Sciences 118. Springer-Verlag, New York, USA (1996).

[7] A. Goldshtein, M. Shapiro and C. Gutfinger, Mechanics of colisional motion of granular materials. Part 3: Self similar shock wave propagation. J. Fluid Mech. 316 (1996) 29-51.

[8] P.S. Gough, Modeling of two-phase flows in guns. AIAA 66 (1979) 176-196.

[9] V. Guillemaud, Modélisation et simulation numérique des écoulements diphasiques par une approche bifluide à deux pressions. Ph.D. Thesis, Université Aix-Marseille I, France (2007).

[10] A. Harten, P.D. Lax and B. Van Leer, On upstream differencing and Godunov-type schemes for hyperbolic conservation laws. SIAM Rev. 25 (1983) 35-61.

[11] J.-M. Hérard and O. Hurisse, A simple method to compute standard two-fluid models. Int. J. Comput. Fluid Dyn. 19 (2005) 475-482.

[12] A.K. Kapila, R. Menikoff, J.B. Bdzil, S.F. Son and D.S. Stewart, Two-phase modeling of deflagration-to-detonation transition in granular materials: reduced equations. Phys. Fluids 13 (2001) 3002-3024.

[13] K.K. Kuo, V. Yang and B.B. Moore, Intragranular stress, particle-wall friction and speed of sound in granular propellant beds. J. Ballistics 4 (1980) 697-730.

[14] J. Nussbaum, Modélisation et simulation numérique d'un écoulement diphasique de la balistique intérieure. Ph.D. Thesis, Université de Strasbourg, France (2007).

[15] J. Nussbaum, P. Helluy, J.-M. Hérard and A. Carriére, Numerical simulations of gas-particle flows with combustion. Flow Turbulence Combust. 76 (2006) 403-417.

[16] V.V. Rusanov, The calculation of the interaction of non-stationary shock waves with barriers. Ž. Vyčisl. Mat. i Mat. Fiz. 1 (1961) 267-279.

[17] R. Saurel and R. Abgrall, A multiphase Godunov method for compressible multifluid and multiphase flows. J. Comput. Phys. 150 (1999) 425-467.

[18] E.F. Toro, Riemann-problem based techniques for computing reactive two-phase flows, in Proc. Third Intl. Conf. on Numerical Combustion, A. Dervieux and B. Larrouturou Eds., Lecture Notes in Physics 351, Springer, Berlin, Germany (1989) $472-481$. 